\title{
Facilitating the conservation
} treatment of Eva Hesse's Addendum through practice-based research, including a comparative evaluation of novel
cleaning systems

Angelica Bartoletti ${ }^{1,2}$, Tamar Maor $^{1,3}$, David Chelazzi ${ }^{4}$, Nicole Bonelli ${ }^{4}$, Piero Baglioni ${ }^{4}$, Lora V. Angelova ${ }^{1,5}$ and Bronwyn A. Ormsby ${ }^{* *}$

\begin{abstract}
This paper describes the methodology and practice-based research underpinning the development of a successful cleaning strategy for Eva Hesse's sculpture Addendum (1967, Tate Collection T02394). Research strands included: technical and art historical investigations to determine the materials and construction of the work of art and to define the aims of the conservation treatment; the production, soiling and accelerated ageing of mock-up samples using contemporary equivalent materials; and the systematic, iterative evaluation of soiling removal systems, which were further refined for appropriate use on the work of art. The comparative cleaning system evaluation was employed to determine options which offered optimal soiling removal efficacy and posed minimal risk to the work of art. Newly developed Nanorestore Gel ${ }^{\circledR}$ Peggy series (i.e. polyvinyl alcohol (PVA) and polyvinyl alcohol/polyvinylpyrrolidone (PVA/PVP)-based hydrogels), designed for the cleaning of modern and contemporary art, were evaluated with a range of other gels, emulsifiers and cosmetic sponges and assessed through a combination of empirical observation, microscopy and spectroscopic techniques. Promising options, combined with tailored aqueous phases derived from trials on mock-up samples, were then evaluated on discreet areas of the sculpture. After extensive testing, the top papier mâché section of Addendum was surface cleaned using an aqueous solution applied with cosmetic sponges, and the ropes were surface cleaned using a modified version of Nanorestore Gel ${ }^{\circledR}$ Peggy 5 (PVA/PVP) loaded with a tailored aqueous solution. The optimisation of this hydrogel, combined with the extensive supporting research, enabled the successful, low-risk, conservation treatment of Addendum for the first time since acquisition.
\end{abstract}

Keywords: Cleaning, Evaluation, Hydrogel, Papier mâché, Acrylic, Polyvinyl acetate, Hesse, Peggy Nanorestore

\section{Introduction}

The removal of surface dirt (or soiling), grime and other marks from modern painted surfaces can prove challenging for many reasons including determining the significance of any soiling layer, devising a low-risk method of

\footnotetext{
${ }^{*}$ Correspondence: bronwyn.ormsby@tate.org.uk

${ }^{1}$ Conservation Department, Tate, Millbank, London SW1P 4RG, UK

Full list of author information is available at the end of the article
}

removal should the decision to remove the soiling layer be taken, and ensuring the cleaning treatment is appropriate within the artists' oeuvre and known aesthetics [1-3]. Other challenges are inherent to the material choices made by the artist, for example, since the mid20th century, several of the paints and coatings employed by artists (and conservators) exhibit inherent softness and solvent-sensitivity; the substrates of the work may be varied; many works intentionally lack traditional forms of
Springer Open

(c) The Author(s) 2020. This article is licensed under a Creative Commons Attribution 4.0 International License, which permits use, sharing, adaptation, distribution and reproduction in any medium or format, as long as you give appropriate credit to the original author(s) and the source, provide a link to the Creative Commons licence, and indicate if changes were made. The images or other third party material in this article are included in the article's Creative Commons licence, unless indicated otherwise in a credit line to the material. If material is not included in the article's Creative Commons licence and your intended use is not permitted by statutory regulation or exceeds the permitted use, you will need to obtain permission directly from the copyright holder. To view a copy of this licence, visit http://creativeco mmons.org/licenses/by/4.0/. The Creative Commons Public Domain Dedication waiver (http://creativecommons.org/publicdomain/ zero/1.0/) applies to the data made available in this article, unless otherwise stated in a credit line to the data. 


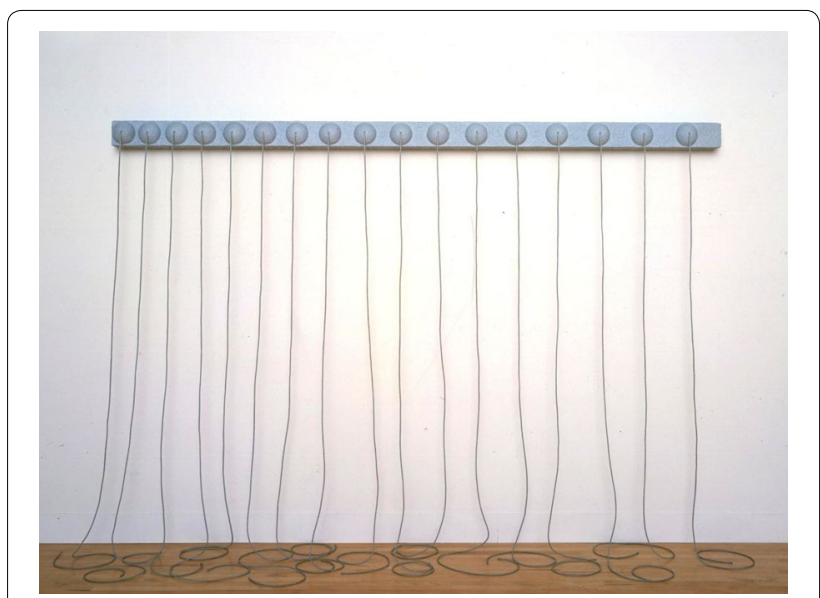

Fig. 1 Eva Hesse, Addendum (1967) Papier mâché, wood and cord; dimensions: a top section: $310 \mathrm{~cm} \times 15 \mathrm{~cm}$ b ropes: approximately $300 \mathrm{~cm}$ long and $6 \mathrm{~mm}$ diameter. Tate collection, T02394 Photo, Tate 2016, before treatment $\odot$ The estate of Eva Hesse, courtesy Hauser \& Wirth, Zürich

protection such as frames and glazing, particularly sculpture, and in some cases, the display context for a given work can prove challenging to the ideals of preservation [4].

One significant example is the sculptural wall installation Addendum (Fig. 1), created in 1967 by the Germanborn American sculptor Eva Hesse (1936-1970) for the exhibition Art in Series. ${ }^{1}$ The sculpture is comprised of a softwood rectangular box with seventeen raised hemispheres arranged at increasing intervals, covered in papier mâché and painted grey with acrylic dispersion (emulsion) paint and coated in an unpigmented polyvinyl acetate (PVAc) layer. Seventeen strands of cotton rope to an approximate total length of $51 \mathrm{~m}$, similarly painted and polyvinyl acetate coated, emerge from the hemisphere centres and trail downwards to snake onto the gallery floor in unpredictable loops. This setting naturally encourages the differential soiling of the lower portion of each of the ropes, which eventually results in the rope-ends becoming darkened and yellowed (Fig. 2). After her return to New York in 1965, Hesse abandoned the use of colour and shifted towards using shades of grey and black [5]; hence it is likely that Addendum was originally conceived as a matt grey monochrome wall installation. The differential soiling compromises the important monotonal character of this work, ${ }^{2}$ which prompted the desire to address this issue.

\footnotetext{
${ }^{1}$ The Art in Series exhibition was held at Finch College, New York, and opened in November 1967. Addendum was purchased by Victor Ganz from the Fourcade Droll Gallery (New York) in 1972, and then acquired by Tate from Victor Ganz (Grant-in-Aid) in 1979.

2 B. Fer. Personal communication. August 8, 2017.
}

Previous attempts $[6,7]$ had proven challenging due to the degree and tenacity of the soiling across the work, where it appeared more embedded into the painted and coated ropes. During the preceding assessment of this work in 2012-2013, preliminary tests were carried out involving, amongst other options, the application of mineral spirit-based microemulsions [7], which proved effective at removing the embedded soiling. However, these systems were not further investigated due to time constraints and concerns around the possible swelling of the paint and coating layers. The removal of soiling and grime from synthetic polymer painted and coated surfaces can pose particular challenges around the use of water and organic solvents during cleaning, including the risk of swelling, extraction of lower molecular weight components, and the layers becoming more vulnerable to removal [8-11]. Any conservation treatment would therefore need to consider the extent of soiling removal possible, explore the risks to the underlying coating, paint, papier mâché or cotton rope layers, as well as consider how the soiling removal process may affect the appearance and viewer's reading of this early and significant Hesse work.

Over the past decade or more, research efforts have enhanced options and methodologies for the cleaning of modern painted surfaces. This has included the refinement and tailoring of cleaning approaches to specific coating/paint polymer types $[12,13]$ and the introduction of a range of novel systems for use on these often unpredictable and challenging surfaces [13, 14]. The present study, alongside the conservation treatment of Roy Lichtenstein's painting Whaam! carried out as part of the same project [15], contributes to this ongoing area of investigation through rigorous practice-based research, primarily focused into an extensive comparative study of a range of both established and novel soiling removal systems.

This case study methodology was designed to include the various supporting research strands as well as the completed conservation treatment; hence, the results are presented iteratively and reflect the conservation treatment decision-making process as it progressed. As part of the process, the sculpture underwent a full art historical investigation, which revealed detailed information on the inspiration and making of the work [16], and a technical and analytical examination (Table 1), which informed the production of mock-up samples, cleaning system selection and the desired treatment outcomes. 

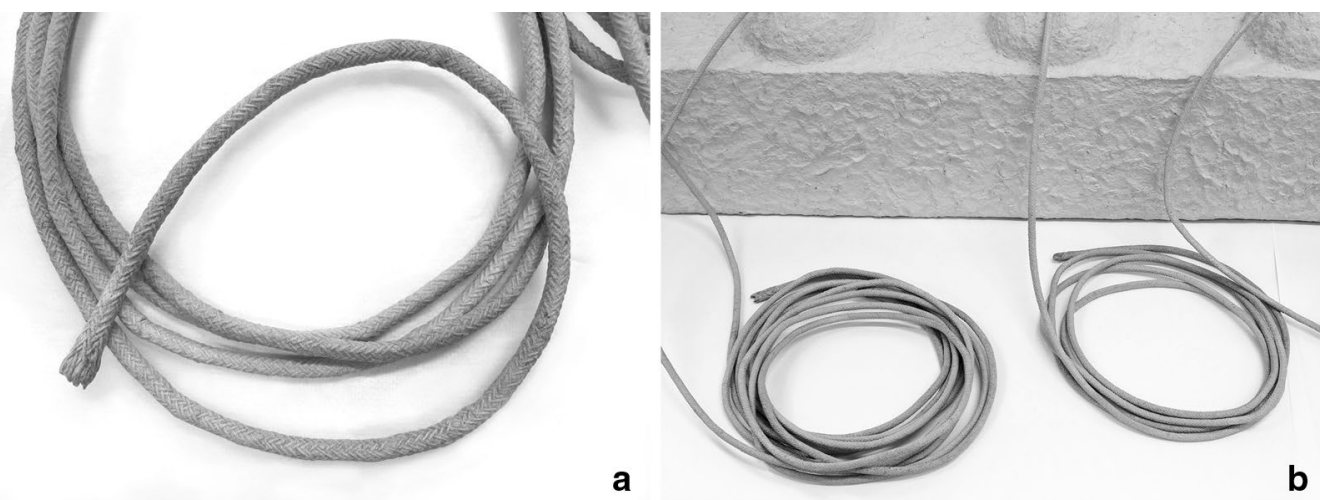

Fig. 2 Details of Addendum showing the level of soil deposited on the rope surface (a), and the discrepancy in tonality between the papier mâché section and the ropes (b). Photo Tate 2017, @ The estate of Eva Hesse, courtesy Hauser \& Wirth, Zürich

\section{Addendum - technical examination}

The top section of the sculpture is constructed from an open box made of wooden boards pasted together with a polyvinyl acetate (PVAc) adhesive, and rubber balls cut in half and pasted on top of the box, to form the hemispheres (Table 1). The entire wooden structure was then covered with a highly-textured pulp-based papier mâché layer (mixed with PVAc glue), painted with two applications of grey paint: a dark grey paint as the lower layer, with a light grey paint on top. The paint binding media was characterised as a poly ethyl acrylate-methyl methacrylate [p(EA-MMA)] acrylic dispersion copolymer containing titanium white (PW6) and Mars black pigments (PBk11), with gypsum as an extender. On top of the grey paint layers, an unpigmented polyvinyl acetate (PVAc) coating is also present.

The cotton ropes, which have weight and diameter similar to sash-window cords, are similarly painted (though with only one light grey paint layer), and PVAc coated. At close inspection, the presence of glossy, discoloured areas was visible on the ropes, which had a distinctive ultraviolet (UV) fluorescence when compared to the rest of the sculpture, as shown in Fig. 3. Further analysis revealed that the ropes have an additional unpigmented coating identified as an acrylic poly $n$-butyl acrylate-methyl methacrylate [p(nBA-MMA)] acrylic dispersion copolymer, which was not previously detected. It is probable that the PVAc coatings are artist applied, perhaps to create a uniform sheen across the entire sculpture; and after some investigation, it appears possible that the upper $\mathrm{p}(n \mathrm{BA}-\mathrm{MMA})$ dispersion coating may also have been artist-applied [16], as the availability of this copolymer type can be traced back to $\sim 1967-1968 .^{3}$

${ }^{3}$ T. Learner, M. Keefe, S. Croll, M. Golden. Personal communication. August 10-11, 2017.
The primary objectives of the conservation treatment were to reduce the amounts of soiling across the whole work, which would decrease the risk of the soil becoming further embedded with time, and to reduce the considerable tonal discrepancy between the upper section and the ropes. This was to be achieved without disturbing the varied surface textures across the work; swelling or displacing the fibres of the ropes; and without affecting the applied coatings which were often (and particularly in the areas touching the floor) uneven, worn and friable. Figure 4 shows details of the ropes, including thin paint layers, visible fibres (Fig. 4a), cracks in the paint layers (Fig. 4b) and a general yellowing of the surface.

\section{Experimental \\ Mock-up sample preparation}

Three different mock-up types were prepared to simulate both the papier mâché and rope sections as described in Table 1 (Additional file 1: Fig. S1). The papier mâchébased replicas were fabricated by soaking newspaper in water overnight, which was then broken down by hand to create a paper pulp and hung in netting to extract as much water as possible before adding PVAc glue [Cléopâtre, Amazon, UK]. The mixture was then hand-pressed and pinched onto wooden boards to achieve the same surface texture as the sculpture. Three samples representing the sculpture hemispheres were made using overturned bowls covered in cling film, followed by pressing the paper pulp on top. For the rope mock-ups, $10 \mathrm{~m}$ of a 16-strand plaited cotton sash cord [James Lever Ropes \& Twines, $\left.\mathrm{UK}^{4}\right]$, were cut into smaller pieces of $8 \mathrm{~cm}$ each. Once the mâché-based mock-ups were completely dry, each replica was painted with two layers of a grey

\footnotetext{
${ }^{4}$ http://www.jameslever.co.uk/index.html (accessed 12.12.19).
} 


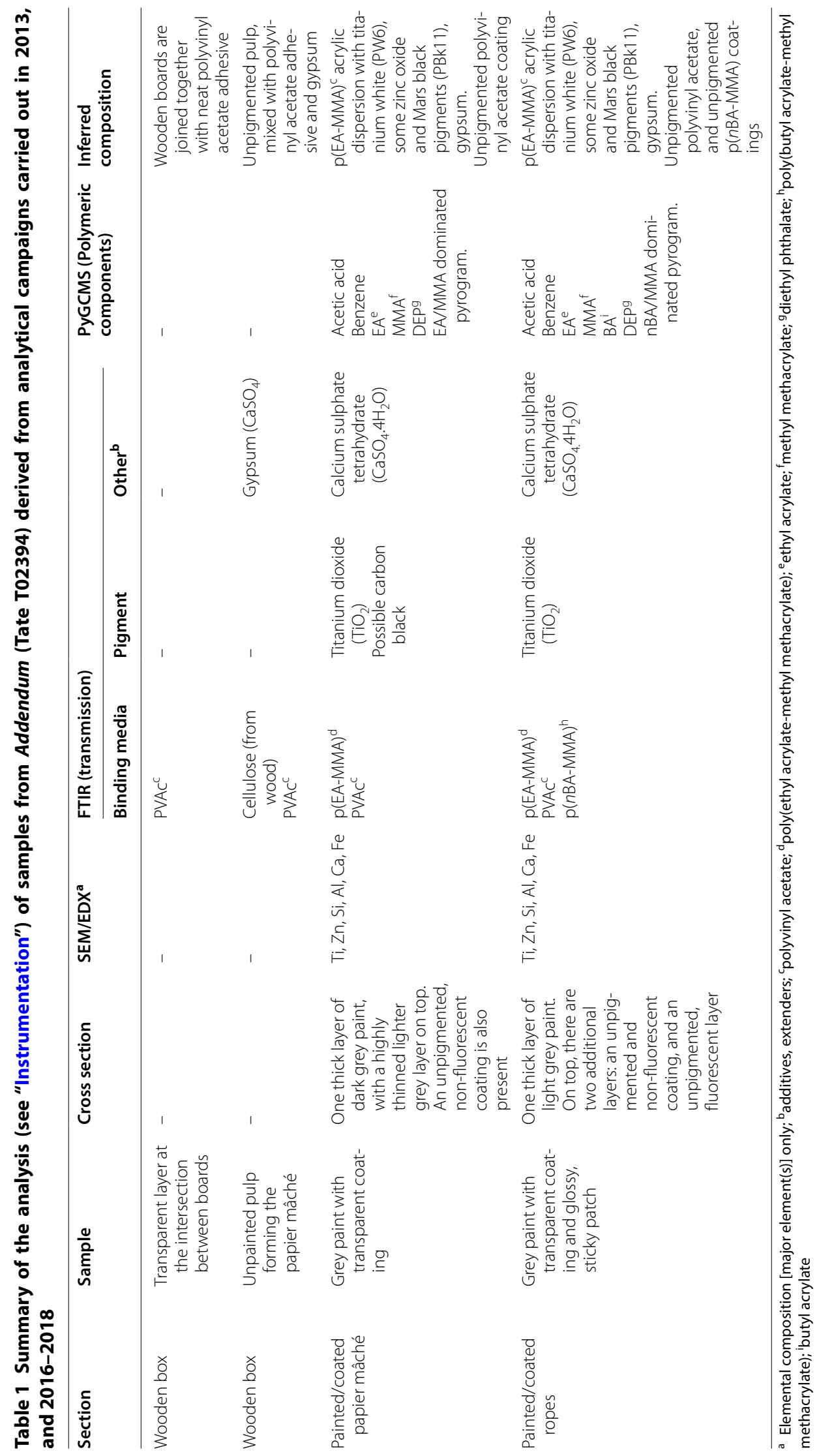



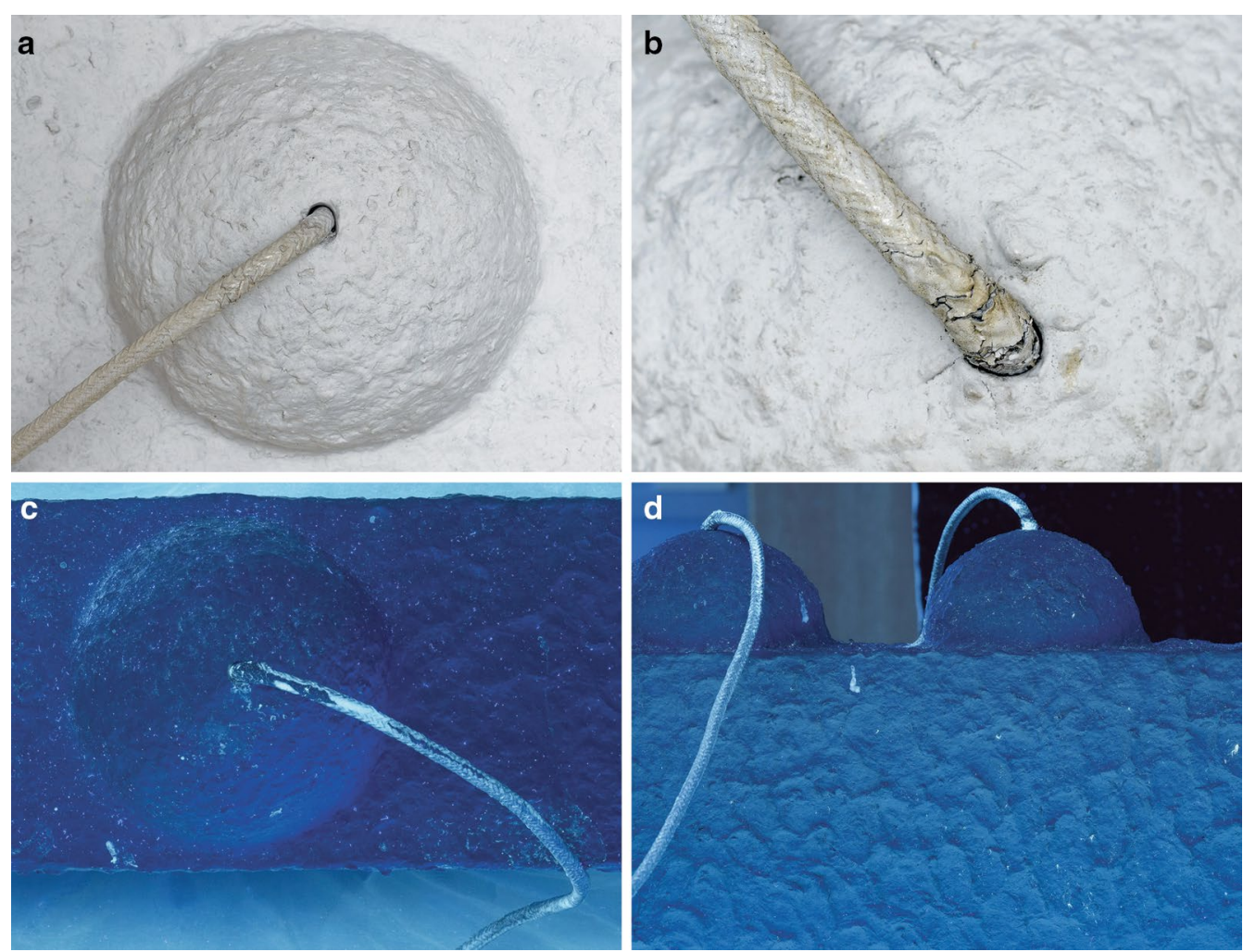

Fig. 3 Details of the sculpture showing the presence of glossy, discoloured areas on top of the ropes ( $\mathbf{a}$ and $\mathbf{b}$, in normal light), which present a distinctive fluorescence compared to the rest of the sculpture (c and $\mathbf{d}$, in ultraviolet light). Photo Tate 2017, ๑ The estate of Eva Hesse, courtesy Hauser \& Wirth, Zürich
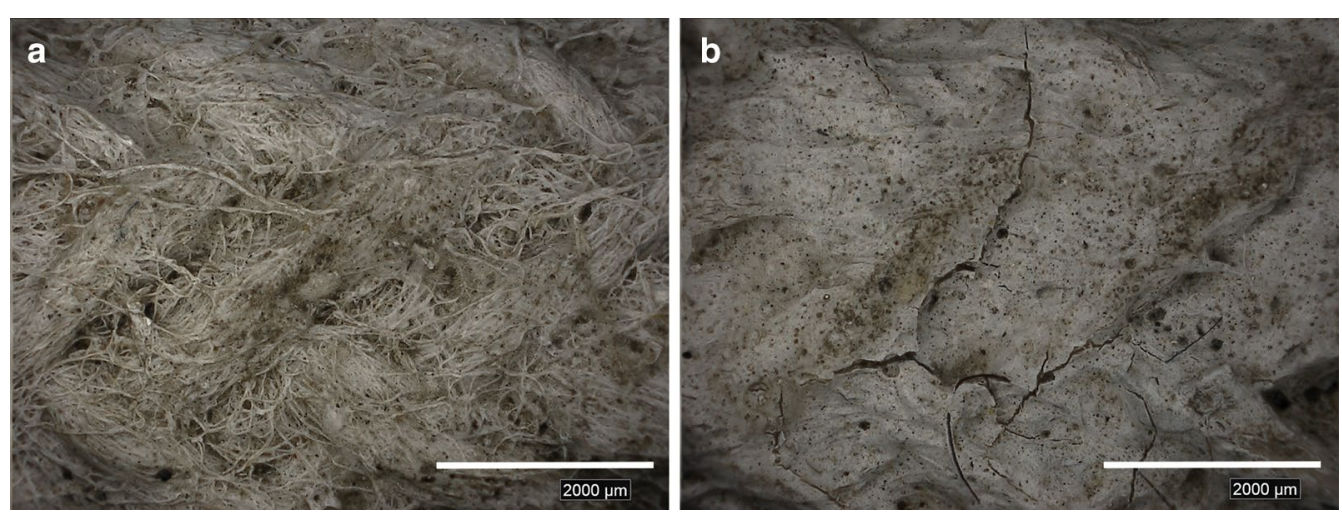

Fig. 4 High-resolution digital (HIROX) images of a rope showing details such as thin paint layers, visible fibres (a), cracks in the coating/paint layers (b), general yellowing of the surface and embedded soil. The scale bar is $2 \mathrm{~mm}$, and the magnification 50x. Photo Tate 2017, @ The estate of Eva Hesse, courtesy Hauser \& Wirth, Zürich

p(EA-MMA) acrylic dispersion paint [Talens Rembrandt Acrylic Colours, Amazon UK]. Graduated amounts of lamp black (PBk7) paint were added to titanium white (PW6) and painted out; when dry, these were compared to the sculpture for colour matching. After several days drying in ambient conditions, the samples were aged in 
a bespoke lightbox, using Phillips daylight tubes with UV filters (average of $10,000 \mathrm{~lx}$ ) at $35^{\circ} \mathrm{C}$ and relative humidity of $30 \% \mathrm{RH}$ for $7^{5}$ days before a layer of PVAc resin [Cléopâtre, Amazon UK] was applied. Samples were aged under the same conditions for another 7 days, and then the additional $\mathrm{p}(n \mathrm{BA}-\mathrm{MMA})$ dispersion layer $\left[\right.$ Primal $^{\circledR}$ AC 35, Kremer Pigmente, Germany] was applied onto the ropes and similarly aged (i.e. for other 7 days). Ageing conditions were adjusted based on those previously used for the natural and accelerated ageing of acrylic-based artist's media [17-19].

The mock-ups were then soiled using a modified version of an artificial indoor particulate soil ${ }^{6}[20]$ applied using an airbrush [BA2503 Basic Spray Gun Set, The Hobby Company LTD, Amazon UK]. The samples were then further aged (for another 20 days) to facilitate the embedding of the artificial soiling into the coating layers, approximating the tenacity of the soil on the sculpture ropes. One papier mâché board and two rope mock-ups were left unsoiled as controls.

\section{Evaluation methodology}

To achieve the treatment aims, a range of established and novel cleaning systems used/designed for modern painted surfaces were selected, and mock-up samples were prepared, aged and soiled using contemporary equivalent materials to act as test substrates as described. This facilitated the trialling and optimising of various systems on the range of materials presented by Addendum and the opportunity to become familiar with the handling and use of novel materials. Once a selection of free-liquid cleaning options had been determined, trials focused on assessing liquid-confining materials which were evaluated empirically and analytically. A selection were then taken forward for limited, discreet trials on Addendum which prompted a final optimisation phase prior to the completion of the conservation treatment.

Designing the optimal cleaning strategy for Addendum involved four phases:

\footnotetext{
5 The art-historical and technical examination of Addendum and research on Eva Hesse's practice and materials selection suggested that all the paint and coating layers present on the sculpture were artist applied, as described by Maor et al. 2020 [16]. It was also assumed that Eva Hesse had applied them at short time intervals. From this, it was decided to subject the mock-up samples to short ageing periods (i.e. 7 days) between the different paint/coating layers application, before applying the artificial soiling. After ageing, the mock-up samples did not fully mimic the sculpture and in particular, the soil was not as embedded as on Addendum. However, the mock-up samples proved useful for becoming familiar with the handling and use of novel materials and for initial soiling removal efficacy trials.

6 The artificial soil mixture consists of iron oxide, silica, kaolin, carbon black, cement type I, gelatine powder, soluble starch, olive oil and mineral oil, suspended in Shellsol ${ }^{\circledR}$ D40 mineral spirits [Kremer Pigmente Germany]. For this study, the carbon black and oil were reduced by approximately $50 \%$.
}

1. Extensive trials of free-liquid systems (listed in Table 2) carried out on mock-up samples. For each test, a hand-rolled cotton swab was dipped into the cleaning solution, and excess liquid was dried off onto a paper towel; the swab was then rolled using consistent light pressure over an area of approximately $1 \mathrm{~cm}^{2}$ up to 10 times. When required, a clearance step was performed with water at $\mathrm{pH} 6$ and conductivity $6 \mathrm{mS} / \mathrm{cm}$ (hereafter referred to as adjusted water 6:6) for aqueous-based solutions, and with the appropriate solvent for the mineral-spirits based solutions and microemulsions. Cleaned areas were assessed straight after testing (including the clearance step when required), and the sample surface had dried. General observations and comments were recorded on the relative cleaning efficacy and intrinsic risks related to each system (such as pigment/ coating removal swelling or changes to the surface).

2. A selection of the most promising free liquids were discreetly applied to Addendum as described above, to explore cleaning efficacy and other parameters. At this stage, the cleaned areas were evaluated using a series of empirical observations (described further in Additional file 1: Table S1). The selected empirical criteria included: dirt removal efficacy (i.e. how soil-free the surface appears after cleaning and clearance steps); pigment pickup (assessed by inspecting the cotton swab after use); surface integrity (judging the dry surface by eye after cleaning relative to the unsoiled control); swelling/blanching noted (as judged by eye and/or using microscopy). For each parameter of interest, the cleaning fluids were rated on a scale from 1 (inadequate/poor) to 5 (most appropriate); the collected data were then recorded and presented as star diagrams (Excel Radar Charts) adjusted from previous research [15, 21-23], where larger stars represent more suitable systems.

3. Several systems for the confinement of cleaning fluids (i.e. thickeners, emulsifiers and gels) were then evaluated on mock-up samples. These systems were firstly prepared using water (either deionised or adjusted water 6:6), to compare their ease of use and degree of conformation to the papier mâché and rope mock-up surfaces. Subsequently, the most appropriate confining systems for this case study were combined with the optimum cleaning fluids selected from discreet trials on the sculpture. Their performance was assessed based on empirical observations and digital microscopy of the cleaned areas immediately after the surface had dried. Here the list of criteria and empirical observations was modified to capture parameters unique to the various gels and emulsifiers and included: cleaning efficacy, ease of use (applica- 


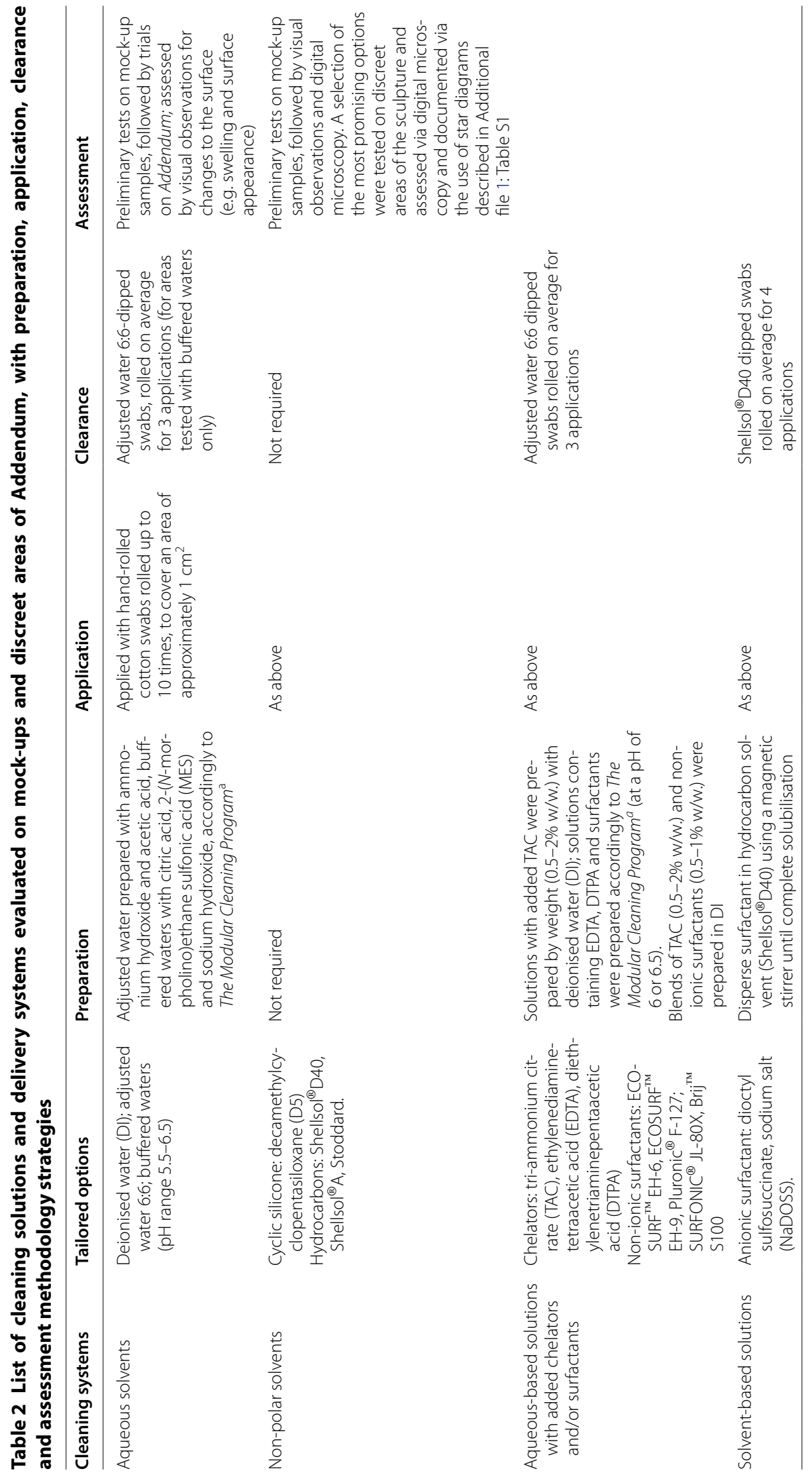




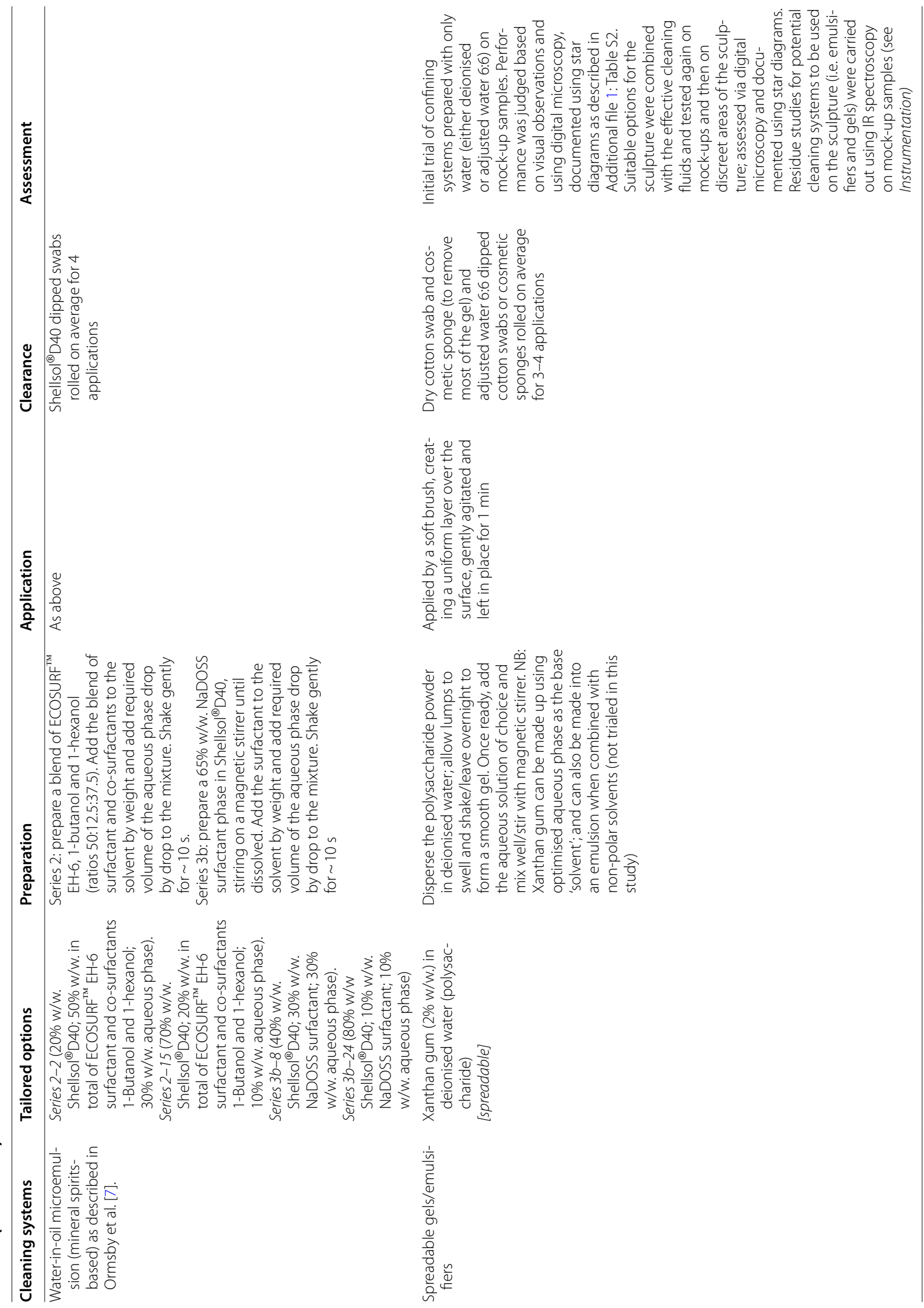




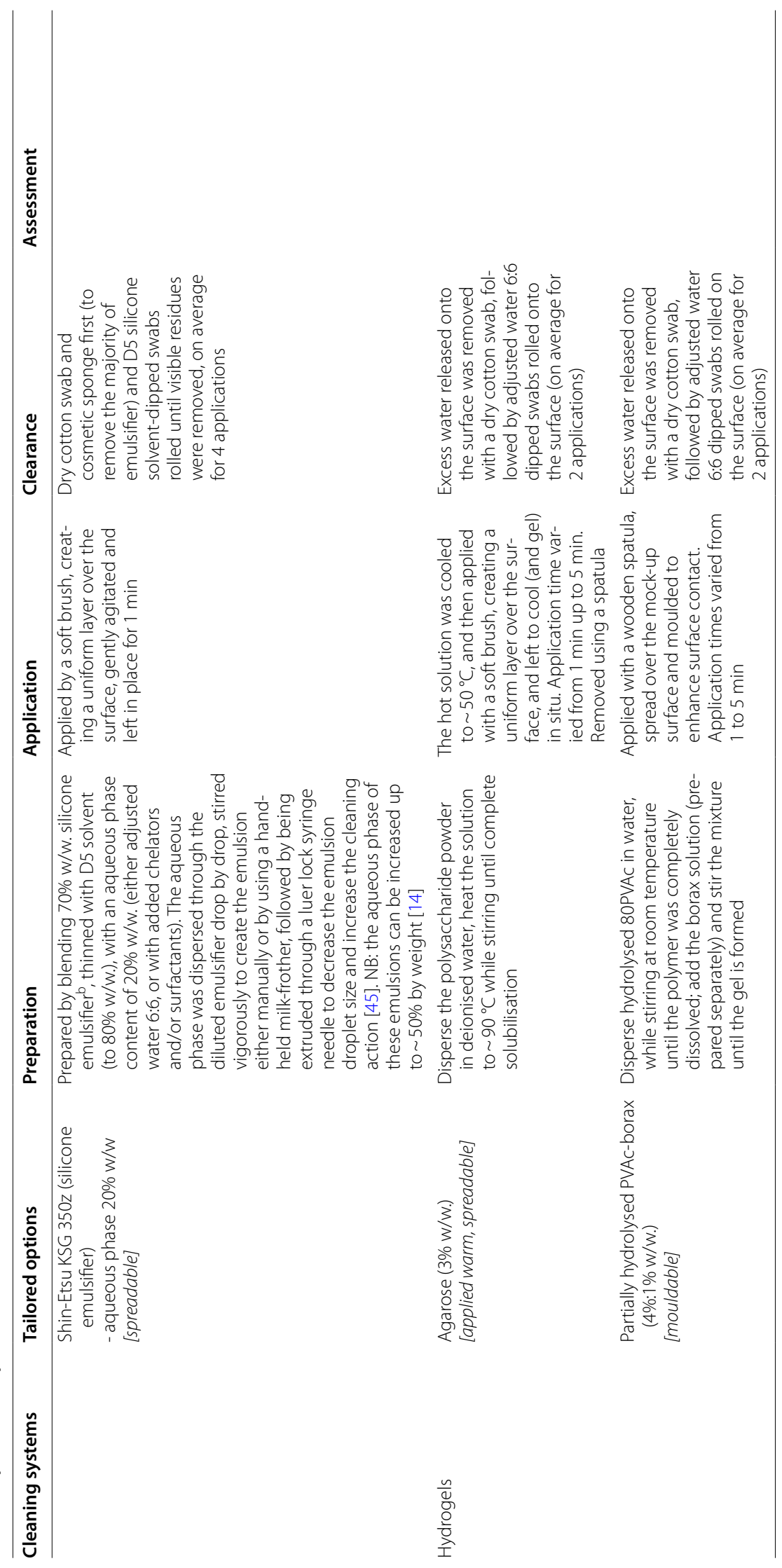




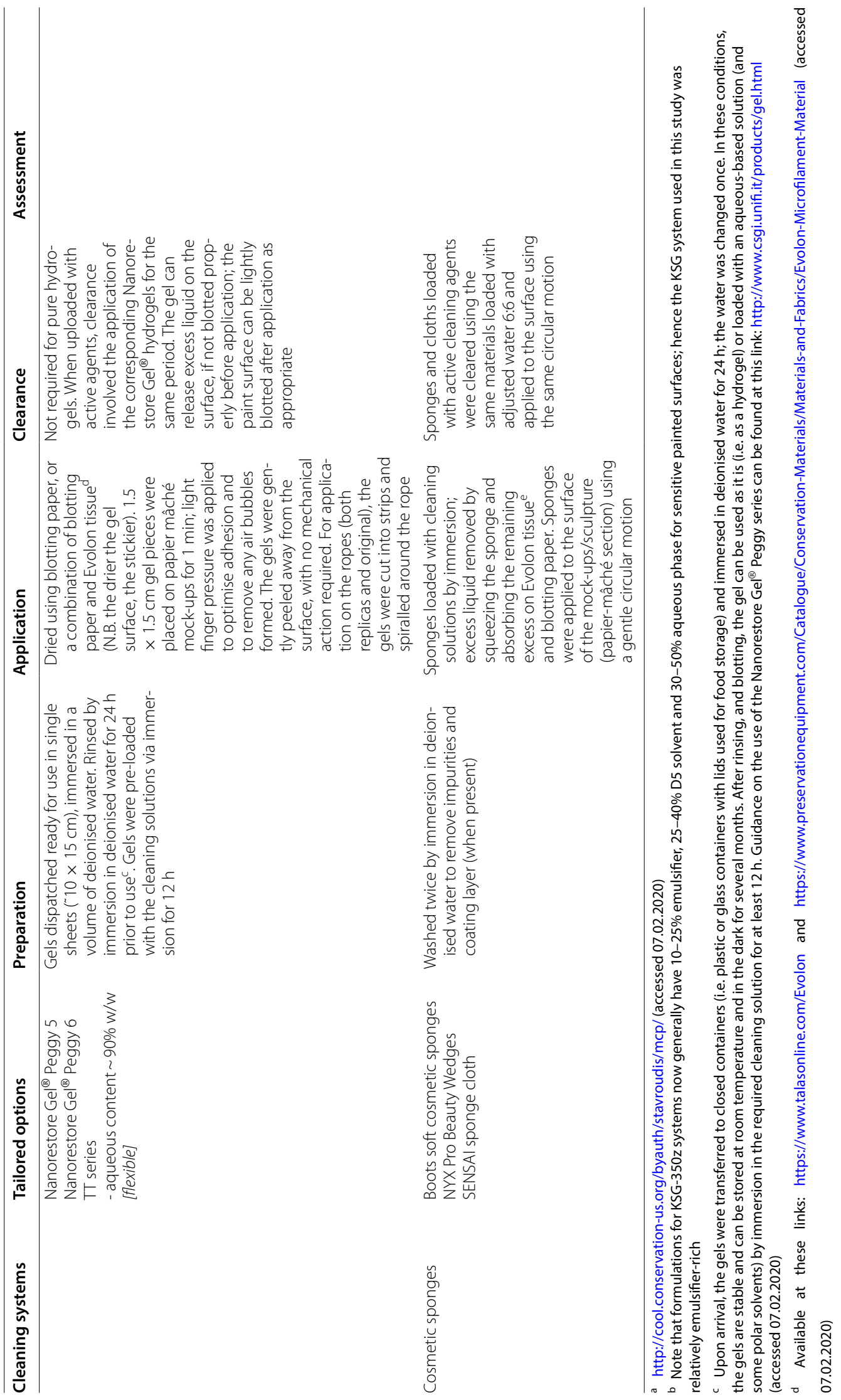


tion, removal and clearance), relative conformation to the surface and relative opacity (as described in Additional file 1: Table S2). This phase also involved modifications to the gels for specific use on Addendum's ropes. Once the promising cleaning options had been identified, studies were carried out on mock-up samples using various forms of IR spectroscopy, to explore the potential presence of emulsifiers, gels and cleaning system residues.

4. The final preparatory phase involved limited, discreet trials on Addendum using a selection of optimised confining systems, to facilitate further refinement prior to commencing the conservation treatment. The cleaned areas were examined using digital microscopy to assess the impact on the sculpture surface and to explore the presence of physical residues.

\section{Cleaning system selection}

Similarly to the conservation treatment on Roy Lichtenstein's painting Whaam! [15], the cleaning materials were chosen from a range of established and novel options to achieve the most appropriate approach(es) for Addendum. Unlike Whaam! [15] which required the use of the same cleaning system across the entire painting surface, the varied construction of Addendum afforded the use of different cleaning systems. One key requirement for Addendum was flexibility, so that potential system(s) could be tailored to each area to account for differences in texture, solvent sensitivity and soiling adhesion. Table 2 lists the cleaning systems and application methods used, such as adjusted and buffered waters derived from The Modular Cleaning Program ${ }^{7}$ [12, 24], a range of confining systems (i.e. thickeners, emulsifiers, gels) which can be used to help minimise undesirable changes to artwork surfaces, in addition to novel materials recently introduced through the NANORESTART project. $^{8}$

The aqueous systems evaluated included adjusted waters with a $\mathrm{pH}$ range of 5 to 7 , set to $6 \mathrm{mS} / \mathrm{cm}$ conductivity, as well as $\mathrm{pH}$ buffered aqueous solutions with added chelators and/or non-ionic alcohol ethoxylate surfactants [12, 24]. Hydrocarbon and cyclic silicone solvents were also initially trialled, as were selected mineral spirits-based microemulsions [7] due to the relative success of these materials in earlier trials [6,7]. Various confining systems were explored to control the

\footnotetext{
${ }_{7}$ http://cool.conservation-us.org/byauth/stavroudis/mcp/ (accessed 12.12.19).

8 NANORESTART was a 42-month collaborative research project (20152018) funded under the EU Framework Programme for Research and Innovation Horizon 2020 (grant agreement number646063); http://www.nanor estart.eu (accessed 12.12.19).
}

cleaning action and reduce mechanical stress, particularly on the degraded $\mathrm{p}(n \mathrm{BA}-\mathrm{MMA})$ coating layer on the ropes. The polysaccharide emulsifier Xanthan gum [25, 26] and the silicone emulsifier Shin-Etsu KSG 350z [13, 14, 25, 27] were selected due to their inherent modifiability and rheological properties. Partially hydrolysed poly(vinyl acetate)-borax (PVAc-borax) mouldable gels [28-31] were included due to their ability to conform and maintain a specific shape; and the rigid polysaccharide gel Agarose [32-36] was included due to the possibility of applying this material warm and left to gel in situ. More recently, a series of novel hydrogels known as the Nanorestore $\mathrm{Gel}^{\circledR}$ series, ${ }^{9}$ showing unique cleaning fluid retention capabilities and physical flexibility, form part of a range of materials developed to avoid the limitations of traditional solvent thickeners [37, 38]. The Nanorestore $\mathrm{Gel}^{\circledR}$ Peggy series ${ }^{10}$ was specifically developed within the NANORESTART project for the cleaning of contemporary works of art with textured or irregular surfaces. These gels are opalescent, physical hydrogels based on a poly(vinyl alcohol) polymeric network, synthesised through a freeze-thaw process [39-42]. Among the options available, Nanorestore Gel ${ }^{\circledR}$ Peggy 5 consists of a blend of polyvinyl alcohol (PVA) and polyvinylpyrrolidone (PVP) polymers, where the PVP provides enhanced retention properties [40]. Nanorestore Gel ${ }^{\circledR}$ Peggy 6 is made from PVA alone and is more flexible and elastic than Nanorestore Gel ${ }^{\circledR}$ Peggy 5, with potential for enhanced conformation to moderately textured surfaces $[39,42]$. One other key advantage of this series is the ability to fine-tune their physical and mechanical properties through adjusting the synthesis procedure. During this case study, new gel options were proposed at the latter stages of the evaluation trials to address the specific challenges associated with the cleaning of the 17 ropes. This involved the production of a series of modifications to Nanorestore Gel ${ }^{\circledR}$ Peggy 5, temporarily called TT gels, which were designed to offer a higher degree of tackiness and self-adhesion.

Lastly, soft cosmetic sponges and cloths ${ }^{11}$ were also explored as alternatives to the contained systems [21, $43,44]$. Options sourced locally included soft cosmetic sponges $^{12}$ (polyurethane-based with a polyethylene glycol coating, Boots UK), NYX Pro Beauty Wedges ${ }^{13}$ (Poly

\footnotetext{
${ }^{9}$ http://www.csgi.unifi.it/products/gel.html (accessed 12.12.19).

10 http://www.csgi.unifi.it/products/peggy.html (accessed 12.12.19).

11 The main polymeric composition of the selected cosmetic sponges and cloth was determined via ATR-FTIR and micro-FTIR (see Instrumentation).

12 https://www.boots.ie/boots-cosmetic-sponge-wedges-10267856 (accessed 12.12.19)

13 https://www.nyxcosmetics.com/pro-beauty-wedges/NYX_240.html (accessed 12.12.19).
} 
(butadiene: styrene)-based) and a SENSAI sponge cloth ${ }^{14}$ (polyvinyl formal-based).

\section{Instrumentation \\ Technical examination of Addendum}

FTIR spectroscopy Transmission FTIR spectroscopy was carried out on small samples taken from Addendum to characterise the paint and coating binding media, pigments and extenders using a Thermo Scientific Nicolet iN10 MX microscope, with a single diamond cell. 64 scans were collected at a resolution of $4 \mathrm{~cm}-{ }^{1}$ across a 4000 to $600 \mathrm{~cm}^{-1}$ range, and data were processed using Omnic 9 software.

Energy-dispersive X-ray analysis (SEM/EDX) SEM/EDX elemental analysis was carried out on cross-sections of the coating and paint layers taken from Addendum, with a LEO 1455VP Scanning Electron Microscope (SEM) with INCA software, using back-scattered electron imaging (BSE), $20 \mathrm{kV}, 15-\mathrm{mm}$ working distance, and $100 \mathrm{~Pa}$ air pressure.

Pyrolysis gas chromatography-mass spectrometry (PyGCMS) PyGCMS analysis was carried out on samples taken from Addendum using a CDS Pyroprobe 5000 heated Pt filament pyrolyser (CDS Analytical) and a Varian CP-3800 gas chromatography coupled with a Varian Saturn 2000 mass spectrometer. Samples were injected in split mode (split ratio 1:50). The GC temperature was initially $50{ }^{\circ} \mathrm{C}$ for $2 \mathrm{~min}$, ramped at $10{ }^{\circ} \mathrm{C} / \mathrm{min}$ to $310^{\circ} \mathrm{C}$, with a final hold of $10 \mathrm{~min}$. Total run time: $43 \mathrm{~min}$. Helium gas flow was set at $1.0 \mathrm{~mL} / \mathrm{min}$. Column: Phenomenex Zebron ZB-5 column (30 m length; $0.25 \mathrm{~mm}$ i.d.; $0.25 \mu \mathrm{m}$ film thickness). MS conditions: EI mode (70 eV); scanned 40-399 amu every $0.49 \mathrm{~s}$.

\section{Cleaning evaluation}

Digital microscopy High-resolution digital microscopy was performed on Addendum (on both the top papier mâché and ropes section) and the rope mock-up samples before, during and after cleaning trials, and after the full cleaning treatment using a HIROX KH-8700 microscope (HIROX, Japan) with an MXG-2500REZ revolver zoom lens set at $50 \times$ magnification, using ring light illumination. Images were processed using HIROX software.

FTIR spectroscopy ATR-FTIR (attenuated total reflectance) spectroscopy was carried out to explore cleaning system residues and changes to rope mock-up samples

\footnotetext{
$\overline{{ }^{14} \text { https://www.sensai-cosmetics.com/en/products/skincare/silky_purifying/ }}$ sponge_chief.html (accessed 12.12.19).
}

using a Thermo Scientific Nicolet iZ10 system and germanium ATR crystal. 64 scans were collected at a resolution of $4 \mathrm{~cm}^{-1}$ across a 4000 to $400 \mathrm{~cm}^{-1}$ range and data processed using Omnic 9 software.

Micro reflectance FTIR-2D imaging was carried out on the rope mock-up samples to explore the presence of cleaning system residues and to assess cleaning efficacy. A Cary 620-670 FTIR microscope was used, equipped with a Focal Plane Array (FPA) $128 \times 128$ detector (Agilent Technologies) in reflectance mode, with an open aperture and a spectral resolution of resolution of $8 \mathrm{~cm}^{-1}$ for 128 scans, which was selected to improve the signalto-noise ratio of the reflectance spectra. The background was acquired using a gold plate. For each location, a map of $700 \times 700 \mu^{2}(128 \times 128$ pixels $)$ was produced, with a spatial resolution of $5.5 \mu \mathrm{m}$ (i.e. each pixel dimension is $5.5 \times 5.5 \mu \mathrm{m}^{2}$ ). For each two-dimensional (2D) map, the intensity of characteristic bands of cleaning solutions, emulsifiers and gels, was imaged and the chromatic scale of the maps shows increasing absorbance of the bands as follows (unless reported otherwise): blue $<$ green $<$ yellow $<$ red.

For both the ATR-FTIR bench system and Micro reflectance FTIR-2D imaging, measurements were obtained in triplicate, and spectra were analysed for characteristic absorption bands of the neat cleaning materials evaluated.

\section{Results and discussion-part 1: cleaning system evaluation and optimisation}

Free-liquid trials-mock-up samples and Addendum

The range of aqueous systems evaluated (Table 2) showed moderate cleaning efficacy on the papier mâché mock-ups and no or minimal cleaning efficacy on the rope mock-ups, where only the unbound soiling was removed (Additional file 1: Table S3). The buffered and citrate waters also offered minimal cleaning efficacy, whilst solutions with added EDTA or DTPA and surfactants were more effective on both mock-ups with no undue effects until longer exposures had been applied. The aqueous solutions with TAC alone and with added surfactants produced successful results with respect to cleaning efficacy, and the chelator-surfactant blends partially reduced the embedded soiling. For these tests, the clearance step, performed with adjusted water 6:6, often resulted in more soil being removed, and minimal swelling was observed in some cases. Not surprisingly, the neat hydrocarbon and silicone solvents performed poorly with respect to cleaning efficacy, with minor changes to the mock-up surfaces noted, as described in Additional file 1: Table S3. The D5 solvent tended to spread quickly and the slow evaporation rate delayed assessment of treated surfaces. Not surprisingly, the aromatic hydrocarbon 

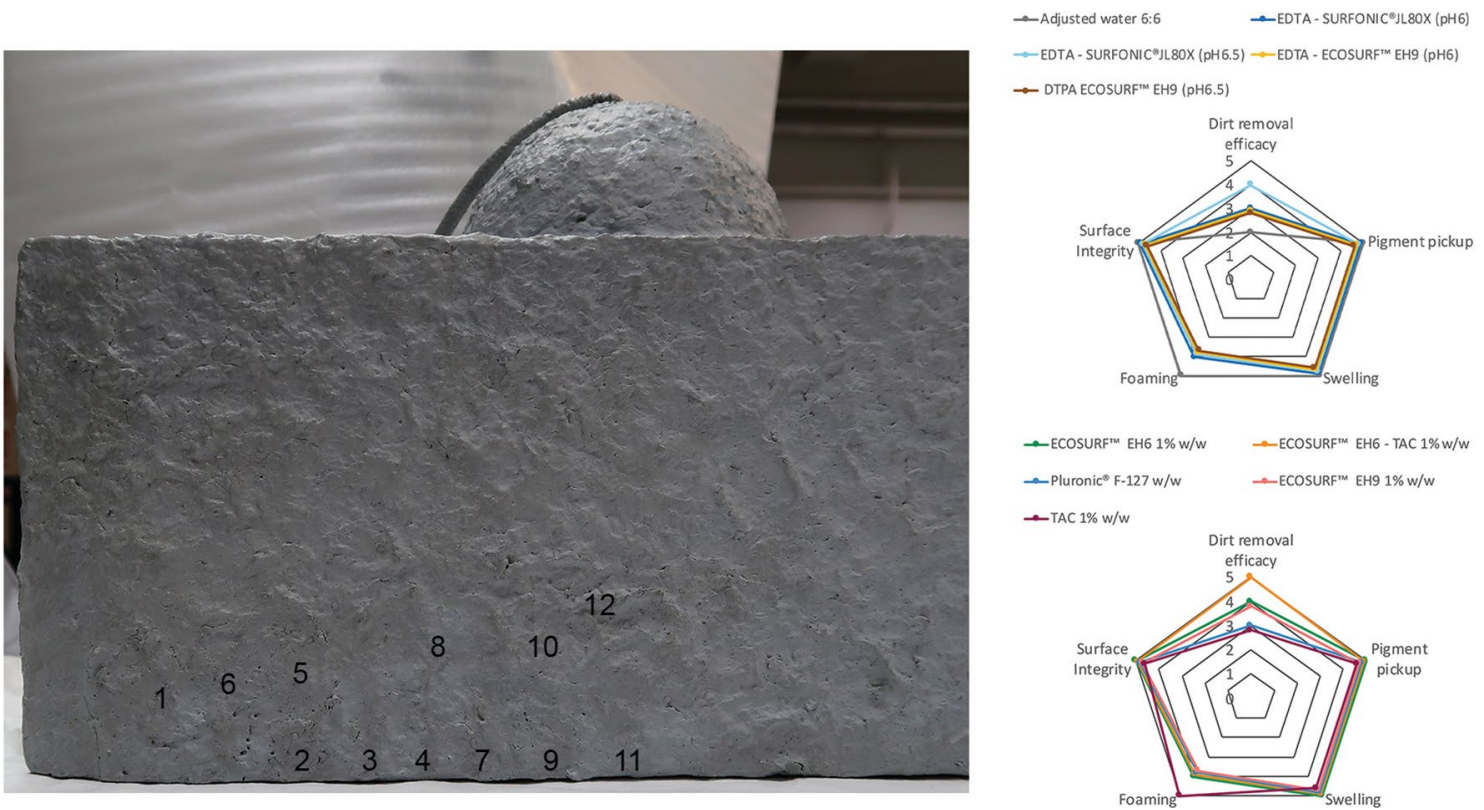

Fig. 5 Preliminary cleaning tests on papier mâché section from Addendum (top side, right corner, viewer's right). Marked tests are: Adjusted water 6:6 (1); $1 \%$ w/w. ECOSURF ${ }^{\mathrm{TM}}$ EH-6 (2); $1 \%$ w/w. ECOSURF ${ }^{\mathrm{TM}}$ EH-6 and 1\% w/w. TAC (3); $1 \%$ w/w. Pluronic ${ }^{\circledR} \mathrm{F}-127$ (4); $1 \%$ w/w. ECOSURF ${ }^{\mathrm{TM}}$ EH $-9(5) ; 1 \%$ W/w. TAC (6); EDTA pH 6 and SURFONIC ${ }^{\circledR}$ JL-80X (7); EDTA pH 6.5 and SURFONIC ${ }^{\circledR}$ JL-80X (8); EDTA pH 6 and ECOSURF ${ }^{\text {TM }}$ EH-9 (9); DTPA pH 6.5 and ECOSURF $^{\mathrm{TM}}$ EH-9 (10). Photo $\odot$ Tate 2017

Shellsol ${ }^{\circledR}$ A disturbed the acrylic dispersion coating on the rope mock-ups, and despite their poor cleaning efficacy, both the D5 and Shellsol ${ }^{\circledR}$ D40 solvents showed promise as non-polar barriers and/or as base solvents for cleaning systems. The Shellsol ${ }^{\circledR} \mathrm{D} 40$-based microemulsions were included in the free-liquid section as they behave as free-liquids, and proved very effective in removing the soiling from the mock-ups (Additional file 1: Table S3); however, except for Series 2-15, the microemulsions tended to alter the surface gloss of the papier mâché and rope mock-ups, suggesting that the coating layers were softening to an unacceptable degree.

The most promising options from the mock-up evaluations (Additional file 1: Table S3) were taken forward for discreet testing on areas of Addendum. Cleaned areas were assessed using rated empirical observations (Additional file 1: Table S1) and the results obtained for the papier mâché and the ropes sections were translated into star diagrams, as shown in Figs. 5 and 6 respectively. Reports on previous cleaning tests performed on Addendum in 2012-2013 [6, 7] suggested that the bulk of the soiling on the papier mâché section could be satisfactorily removed using adjusted water 6:6. However, during the recent assessment, the same adjusted water showed minimal cleaning efficacy, suggesting perhaps that the soiling layer had become more embedded.
For the papier mâché section (Fig. 5), the TAC solution (at $1 \% \mathrm{w} / \mathrm{w}$.) did not perform as well as the non-ionic surfactant solutions which, when used at $1 \% \mathrm{w} / \mathrm{w}$., produced satisfactory results, with the ECOSURF ${ }^{\mathrm{TM}} \mathrm{EH}-6$ and EH-9 options performing better than Pluronic ${ }^{\circledR}$ F-127. As shown in Fig. 5, the blend of ECOSURF ${ }^{\mathrm{TM}}$ EH-6/TAC proved optimal for this part of the sculpture.

Tests carried out on the sculpture ropes (Fig. 6) confirmed that the coating layer was prone to removal through softening and mechanical action, and that the soiling was significantly more embedded when compared to the mock-up equivalents and the papier mâché section. Here very few of the options trialled on the mock-ups warranted further exploration. Amongst those having some effect, the blend of ECOSURF ${ }^{\mathrm{TM}}$ EH-6/TAC (both at $1 \% \mathrm{w} / \mathrm{w}$.) proved most promising. Hence, further tests were carried out increasing the TAC concentration up to $2 \% \mathrm{w} / \mathrm{w}$., which enhanced the cleaning action and facilitated more even soiling removal.

\section{Gel, emulsifier, and application method selection- mock-up samples}

During this phase, the liquid-confining materials listed in Table 2 were tested as prepared hydrogels (using either deionised or adjusted water 6:6) to assess their inherent cleaning efficacy, ease of use and degree of conformation to the papier mâché and rope mock-up surfaces (Table 3). 

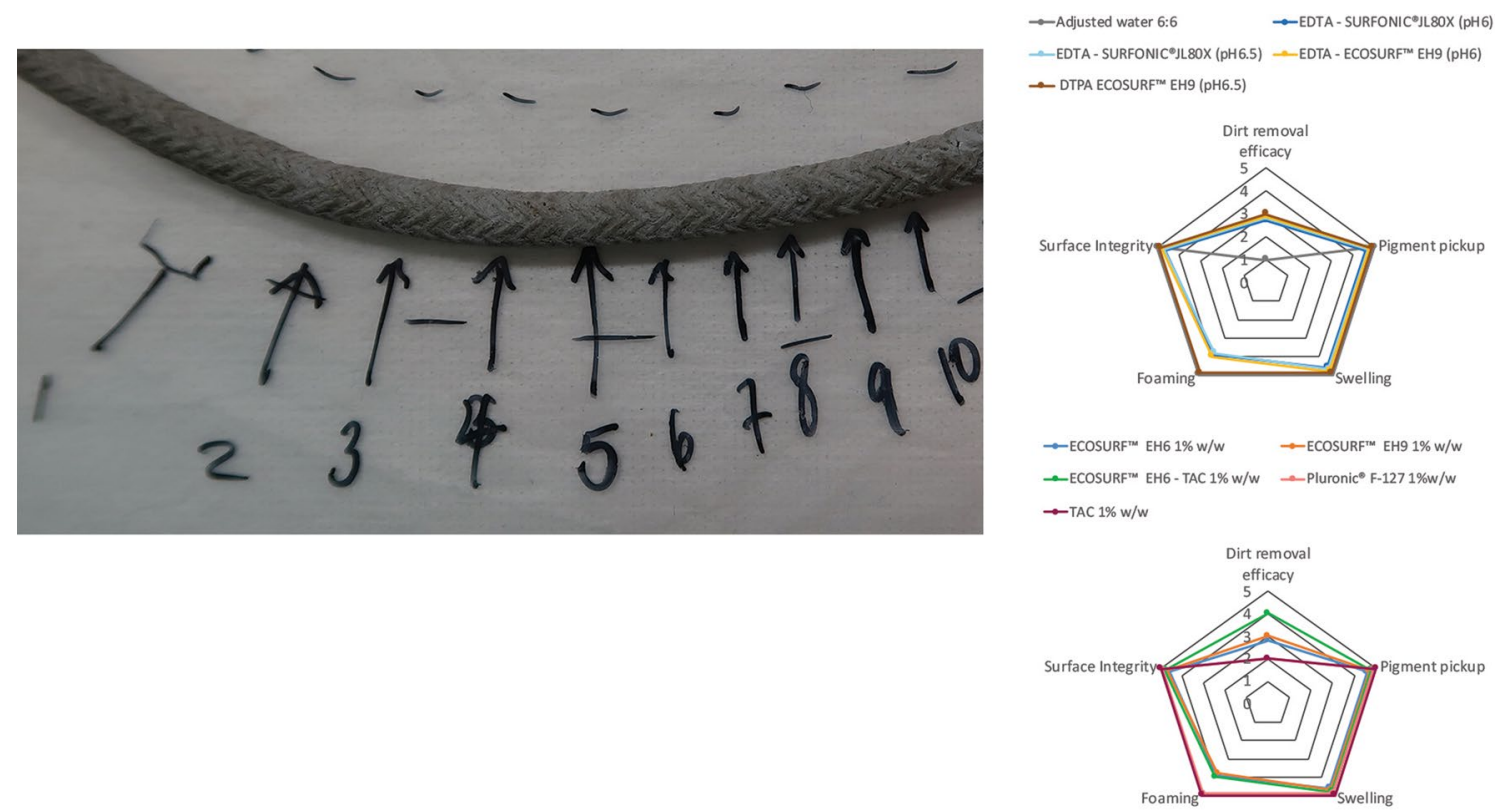

Fig. 6 Preliminary tests on rope number 9 from Addendum. Marked tests are: Adjusted water 6:6 (1); $1 \% \mathrm{~W} / \mathrm{W}$. ECOSURF ${ }^{\mathrm{TM}} \mathrm{EH}-6(2) ; 1 \% \mathrm{~W} / \mathrm{W}$. ECOSURF $^{\mathrm{TM}}$ EH-9 (3); $1 \%$ w/w. ECOSURF ${ }^{\mathrm{TM}}$ EH- 6 and $1 \%$ w/w. TAC (4); $1 \%$ w/w. Pluronic ${ }^{\circledR}$ F-127 (5); EDTA pH 6 and SURFONIC ${ }^{\circledR}$ JL-80X (6); EDTA pH

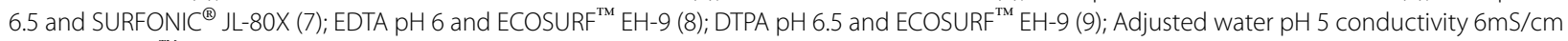
(10); ECOSURF $^{\mathrm{TM}}$ EH-9 at pH 5 (11). Photo Tate $\odot 2017$

The results for the papier mâché mock-ups presented in Fig. 7 suggest that the Agarose gel offered optimal surface contact and that the removal of the gel was straightforward; however, the inherent cleaning efficacy proved minimal. Applications were then increased up to 5-min, where the cleaning efficacy only slightly increased, and water was released onto the surface. Agarose was not tested on the rope mock-ups due to concerns around the temperature required for optimal gel use $\left(\sim 50{ }^{\circ} \mathrm{C}\right)$ and the significantly lower glass transition temperature of the $\mathrm{p}(n \mathrm{BA}-\mathrm{MMA})$ coating $\left(\sim 10-15^{\circ} \mathrm{C}\right)[46,47]$.

The mouldable PVAc-borax gels offered initially promising contact with mock-up surfaces; however, this tended to decrease with time. To promote contact, a glass weight was applied to the gel, with little success on the papier-mâché section, while finger pressure partly enhanced the cleaning efficacy on the ropes. However, as was also observed for the Agarose gel, water was released onto the mock-up surfaces which caused redeposition of the soiling layer across the surface. Hence, this gel was not taken forward for further testing. ${ }^{15}$

\footnotetext{
${ }^{15}$ PVAc-borax gels also present limitations with respect to the maximum concentration of chelators and surfactants required to form successful gels, i.e. up to $0.5 \% \mathrm{w} / \mathrm{w}$.
}

Both of the spreadable systems (Xanthan gum and Shin-Etsu KSG 350z) offered optimal contact with the surface when applied by brush and offered promising soil removal. However, it was noted that these materials necessitated the cleaning of small areas at a time and that the clearance procedures required several applications to remove all visible residues. These factors, when combined with ongoing Health and Safety concerns, ${ }^{16}$ resulted in the ratings shown in Fig. 7.

As listed in Table 3, although the Nanorestore Gel ${ }^{\circledR}$ Peggy 5 and Nanorestore Gel ${ }^{\circledR}$ Peggy 6 show a unique degree of flexibility, in this case, they offered only moderate contact with the mock-up surfaces (Fig. 7); and had a tendency to unroll when applied to the ropes, resulting in an uneven removal of the dirt layer for both mock-up types, with soil remaining in the more textured areas.

Of the two Nanorestore gels, the Nanorestore $\mathrm{Gel}^{\circledR}$ Peggy 5 hydrogel removed more soiling from both mockups; however, Nanorestore Gel ${ }^{\circledR}$ Peggy 6 offered enhanced

\footnotetext{
${ }^{16}$ The use of silicone solvents, such as octamethylcyclotetrasiloxane (D4) and decamethylcyclopentasiloxane (D5), have been restricted by the European Commission in rinse-off cosmetic products with a concentration of $0.1 \%$ or more of either substance, due to their toxicity potential and because they tend to accumulate in the environment with unpredictable long-term effects. https://www.chemsafetypro.com/Topics/Restriction/Restriction_of_D4_ and_D5_in_Personal_Care_Products.html (accessed 12.12.19).
} 
Table 3 Key observations for trials with emulsifiers, gels and cosmetic sponge options, prepared or combined with water only (either deionised or adjusted water 6:6), tested on the mock-up samples

\begin{tabular}{|c|c|c|c|c|}
\hline & \multicolumn{2}{|c|}{ Papier-mâché: [p(EA-MMA) + PVAc] } & \multicolumn{2}{|c|}{ Ropes: $[p(E A-M M A)+P V A c+p(n B A-M M A)]$} \\
\hline & Pros & Cons & Pros & Cons \\
\hline Agarose gel & $\begin{array}{l}\text { Easy application and } \\
\text { removal } \\
\text { Optimal surface contact }\end{array}$ & $\begin{array}{l}\text { Poor inherent surface } \\
\text { cleaning } \\
\text { Release of water onto the } \\
\text { surface }\end{array}$ & Not tested due to rigidity & $\begin{array}{l}\text { Temperature of application } \\
\text { higher than glass transi- } \\
\text { tion temperature of the } \\
\text { uppermost } \mathrm{p}(n \mathrm{BA}-\mathrm{MMA}) \\
\text { coating }\end{array}$ \\
\hline PVAc-Borax gel & $\begin{array}{l}\text { Easy application and } \\
\text { removal } \\
\text { Good initial surface contact }\end{array}$ & $\begin{array}{l}\text { Minimal cleaning efficacy } \\
\text { Loss of contact with the } \\
\text { surface } \\
\text { Not compatible with } \\
\text { surfactant concentrations } \\
\text { higher than } 0.5 \% \mathrm{w} / \mathrm{w}\end{array}$ & $\begin{array}{l}\text { Easy application and } \\
\text { removal } \\
\text { Good initial surface contact }\end{array}$ & $\begin{array}{l}\text { Minimal cleaning efficacy } \\
\text { Loss of contact with the } \\
\text { surface } \\
\text { Not compatible with } \\
\text { surfactant concentrations } \\
\text { higher than } 0.5 \% \mathrm{w} / \mathrm{w}\end{array}$ \\
\hline $\begin{array}{l}\text { Xanthan gum } \\
\text { (gel only) }\end{array}$ & $\begin{array}{l}\text { Optimal surface contact } \\
\text { Moderate inherent cleaning } \\
\text { efficacy } \\
\text { Modifiable activity }\end{array}$ & $\begin{array}{l}\text { Small areas at a time } \\
\text { Several clearance steps } \\
\text { required } \\
\text { Time-consuming }\end{array}$ & $\begin{array}{l}\text { Optimal surface contact } \\
\text { Promising cleaning efficacy } \\
\text { Modifiable activity }\end{array}$ & $\begin{array}{l}\text { Small areas at a time } \\
\text { Several clearance steps } \\
\text { required } \\
\text { Mechanical action required } \\
\text { Time-consuming }\end{array}$ \\
\hline Shin-Etsu KSG350z emulsion & $\begin{array}{l}\text { Optimal surface contact } \\
\text { Moderate inherent cleaning } \\
\text { efficacy } \\
\text { Modifiable activity }\end{array}$ & $\begin{array}{l}\text { Small areas at a time } \\
\text { Several clearance steps } \\
\text { required } \\
\text { Time-consuming } \\
\text { Clearance with silicone } \\
\text { solvents } \\
\text { Requires portable extraction } \\
\text { and personal protective } \\
\text { equipment (PPE) }\end{array}$ & $\begin{array}{l}\text { Optimal surface contact } \\
\text { Promising cleaning efficacy } \\
\text { Modifiable activity }\end{array}$ & $\begin{array}{l}\text { Small areas at a time } \\
\text { Several clearance steps } \\
\text { required } \\
\text { Mechanical action required } \\
\text { Time consuming } \\
\text { Clearance with silicone } \\
\text { solvents } \\
\text { Requires portable extraction } \\
\text { and PPE }\end{array}$ \\
\hline $\begin{array}{l}\text { Nanorestore Gel }{ }^{\circledR} \text { Peggy } \\
5 \text { gel }\end{array}$ & $\begin{array}{l}\text { Easy application and } \\
\text { removal } \\
\text { Promising cleaning efficacy } \\
\text { Modifiable activity }\end{array}$ & $\begin{array}{l}\text { Moderate surface contact } \\
\text { Uneven cleaning efficacy }\end{array}$ & $\begin{array}{l}\text { Easy application and } \\
\text { removal, no mechanical } \\
\text { action required } \\
\text { Promising cleaning efficacy } \\
\text { Modifiable activity }\end{array}$ & $\begin{array}{l}\text { Loss of contact with the } \\
\text { surface } \\
\text { Uneven cleaning efficacy }\end{array}$ \\
\hline $\begin{array}{l}\text { Nanorestore Gel }{ }^{\circledR} \text { Peggy } \\
6 \text { gel }\end{array}$ & $\begin{array}{l}\text { Easy application and } \\
\text { removal } \\
\text { Modifiable activity } \\
\text { Better contact than Nanore- } \\
\text { store Gel }{ }^{\circledR} \text { Peggy } 5\end{array}$ & $\begin{array}{l}\text { Moderate surface contact } \\
\text { Uneven cleaning efficacy }\end{array}$ & $\begin{array}{l}\text { Easy application and } \\
\text { removal, no mechanical } \\
\text { action required } \\
\text { Promising cleaning efficacy } \\
\text { Modifiable activity }\end{array}$ & $\begin{array}{l}\text { Loss of contact with the } \\
\text { surface } \\
\text { Uneven cleaning efficacy }\end{array}$ \\
\hline Boots sponge & $\begin{array}{l}\text { Gentle mechanical action } \\
\text { possible, control of } \\
\text { amounts of liquid }\end{array}$ & $\begin{array}{l}\text { Extensive rinsing required } \\
\text { prior to use } \\
\text { Release of large amount of } \\
\text { liquids on the surface }\end{array}$ & Not tested & Not tested \\
\hline NYX Pro Beauty Wedges & $\begin{array}{l}\text { As for Boots sponge. Firm } \\
\text { consistency } \\
\text { No abrasion or resistance } \\
\text { when applied to the } \\
\text { surface }\end{array}$ & $\begin{array}{l}\text { Release of some liquid on } \\
\text { the surface }\end{array}$ & Not tested & Not tested \\
\hline SENSAI sponge cloth & $\begin{array}{l}\text { As for Boots sponge. Very } \\
\text { absorbent } \\
\text { Good control over the } \\
\text { cleaning action } \\
\text { No abrasion or resistance } \\
\text { when applied to the } \\
\text { surface }\end{array}$ & $\begin{array}{l}\text { Release of some liquid on } \\
\text { the surface }\end{array}$ & Not tested & Not tested \\
\hline
\end{tabular}

surface contact. In order to maximise the adhesion between Nanorestore Gel $^{\circledR}$ Peggy 6 and the mock-up surfaces, a series of trials were carried out. For the flat papier mâché mock-ups, the gels were weighted using a conservationweight-bag (476 g), and a home-made weight-bag (165 g), with a Mylar sheet barrier placed between the surface and the weight. Although the contact improved slightly, water deposited onto both the mock-up surface and Mylar sheets suggested that weighting promotes the release of liquid 


\section{Papier mâché mock-ups}

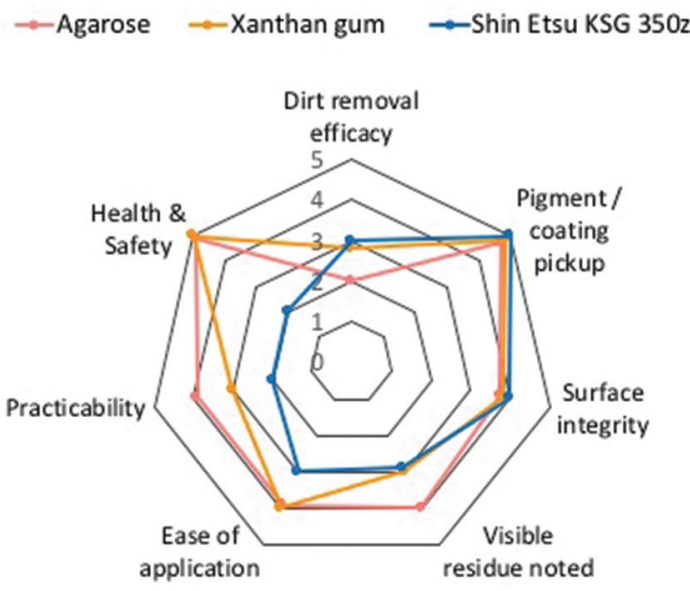

Rope mock-ups

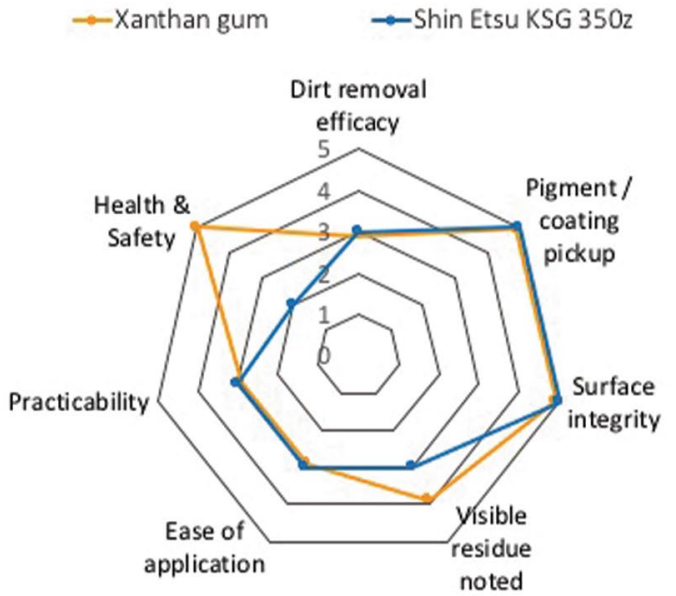

Papier mâché mock-ups

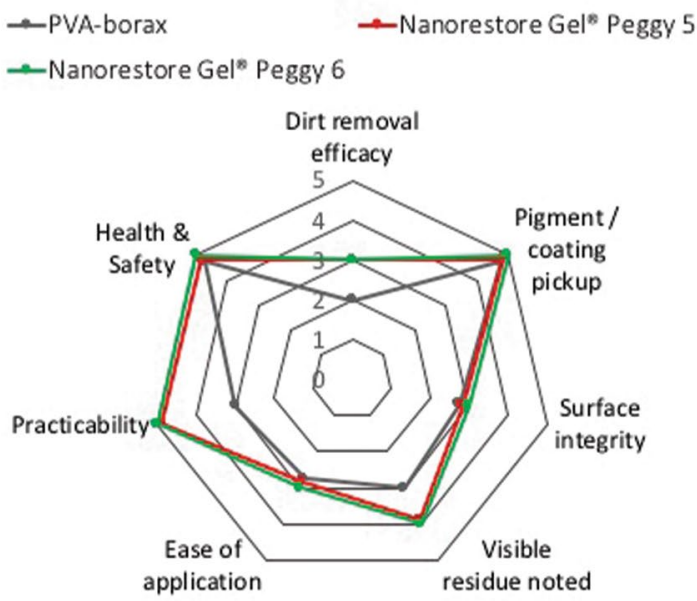

Rope mock-ups

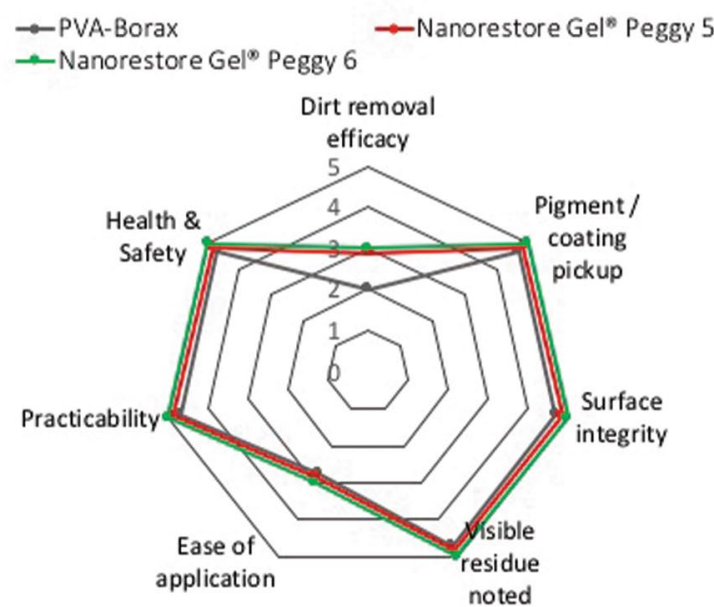

Fig. 7 Star diagrams showing the performance of each of the solvent-containing systems evaluated for the papier mâché (top row) and rope (bottom row) mock-ups, as prepared with only water (either deionised or adjusted water 6:6 as per Table 2). N.B: the exposure time was 5-min for agarose, and 1-min for all other systems. Image ๑ Tate

from this gel. In addition, this approach could not be used on the curved papier-mâché hemisphere shapes.

To explore this further, Nanorestore Gel ${ }^{\circledR}$ Peggy 6 was applied as a spiral, wrapped around the rope profile, where it tended to slowly unroll and detach. To investigate ways of holding the gel in place, cling film was evaluated, with a small clamp applied to hold the ends of the gel in position. A piece of plumbing pipe, cut in half and lined with Plastazote foam and Mylar, was also applied onto the gel and clamped in place for between 1 and $10 \mathrm{~min}$ (Additional file 1: Fig. S2). After these tests, no excess liquid was noted on the rope mock-ups or Mylar, and impressions left in the gel suggested it had conformed to the rope textures. Examination of the cleaned sections revealed no changes to the rope surface topography; though the gel strip proved difficult to place neatly within the pipe section.

For the top section mock-up (Table 3), none of the systems proved optimal. Where the spreadable gels and emulsifiers provided good surface contact, their relatively poor cleaning efficacy and the need for several clearance steps proved less than ideal. In addition, though several attempts were made to enhance the adhesion of the Nanorestore Gel ${ }^{\circledR}$ Peggy 6 to the papier mâché section, it was clear that this gel did not provide optimal contact with the surface.

To explore other options, three soft cosmetic sponges (Tables 2 and 3) were initially tested using adjusted water 6:6 on the flat papier-mâché mock-ups. Among the 
options selected, the Boots sponge proved less favourable (Table 3) due to the need for extensive rinsing to remove a polyethylene glycol coating which rendered the sponge absorbent, but not retentive. The NYX Pro Beauty Wedges offer a firmer consistency and did not drag on the mock-up surface. Lastly, the SENSAI sponge cloth offered greater control over the cleaning action due to its thinness and density; and could be washed and reused. This cloth is also very absorbent, and after blotting, did not release liquid onto the mock-up surface.

\section{Additional PVA-based gels-rope mock-up}

Due to the difficulties experienced with keeping the Nanorestore $\mathrm{Gel}^{\circledR}$ Peggy series in contact with the ropes, a new set of gels, temporarily known as $T T$ gels, were synthesised for further evaluation. This series consisted of adaptations of the Nanorestore Gel ${ }^{\circledR}$ Peggy 5 (PVA/ $\mathrm{PVP})$ prepared by either changing the ratio between the two copolymers, increasing the number of freeze-thaw cycles, and/or the synthesis parameters [40, 42], to offer greater stickiness to aid in wrapping the gels around the ropes so they did not require additional clamping. The TT gels, loaded with deionised water only, were tested on the range of rope mock-ups (Additional file 1: Table S4), to evaluate their suitability for Addendum and compare their physical and mechanical properties with the Nanorestore Gel ${ }^{\circledR}$ Peggy series. After a series of trials, the most promising option proved to be TT14 thin, which when compared to Nanorestore Gel ${ }^{\circledR}$ Peggy 5 and Nanorestore Gel ${ }^{\circledR}$ Peggy 6, was significantly thinner and almost completely transparent. TT14 thin also has a lower storage modulus (data not shown) and, consequently, it is softer and adapted more easily to the rope surfaces. As a result of the adjustments to the synthesis process, TT14 thin was also less retentive than the other Nanorestore $\mathrm{Gel}^{\circledR}$ Peggy series, and therefore required more blotting before application, in this case achieved using blotting paper covered with a layer of Evolon tissue.

\section{Optimising gels and emulsifiers-rope mock-ups}

Further tests were performed on the rope mock-ups using the most promising container systems and freeliquids derived from tests on the sculpture. For this, the blend of ECOSURF ${ }^{\mathrm{TM}} \mathrm{EH}-6 / \mathrm{TAC}$ (1\% and 2\% w/w. respectively) was selected for initial tests, combined with Xanthan gum, Shin-Etsu KSG350z, Nanorestore Gel ${ }^{\circledR}$ Peggy 6 and TT14 thin. At this stage, the Nanorestore $\mathrm{Gel}^{\circledR}$ Peggy 6 gel was applied via clamping as described earlier for between 1 and $5 \mathrm{~min}$. The results (Additional file 1: Fig. S3) illustrate that these systems proved equally efficient in removing the soiling layer and that the TT14 thin gel was the most suitable across all parameters. In this case, the rope mock-ups were easier to clean than the sculpture; however, these trials proved particularly useful for acquiring dexterity with the different emulsifiers and gel systems.

\section{Cleaning system residue evaluation-mock-ups}

Before proceeding with the final tests on Addendum, the rope mock-ups were used to investigate the possible presence of cleaning system residues (gel, chelator etc.) on surfaces after cleaning and clearance. The study was carried out on the options deemed suitable for Addendum's ropes, i.e. Xanthan gum, Shin-Etsu KSG-350z silicone emulsifier and TT14 thin hydrogel. The mock-ups were initially investigated using an ATR- FTIR system (see "Instrumentation") via analysing a control unsoiled area, a control soiled area and areas cleaned with the most promising sets of emulsifiers and gels. In all cases no residues were detected. These investigations were repeated using microFTIR-2D imaging which has lower detection limits $\left(<0.6 \mathrm{pg} /\right.$ pixel, 1 pixel $=30.25 \mu^{2}$, as measured for PVA and PVP films on metallic $\mathrm{Al}$ surfaces) [40]. The presence of residues were explored via mapping characteristic absorption bands from each cleaning material, such as the axial deformation of $\mathrm{C}=\mathrm{O}$ of enols ( $\beta$-diketones) at $1530-1650 \mathrm{~cm}^{-1}$ for Xanthan gum; the silicone-methyl stretching band at $\sim 1250-1260 \mathrm{~cm}^{-1}$ for the Shin-Etsu KSG-350z silicone emulsifier; the $\mathrm{C}=\mathrm{O}$ stretching band of PVP at $1620-1670 \mathrm{~cm}^{-1}$ for the TT14 thin gel $[40,41]$. The cleaning efficacy of each system (applied as described in Table 2) was also evaluated via mapping the $\mathrm{Si}-\mathrm{O}$ stretching band of kaolin, one of the constituents of the artificial soiling mixture, in the range between 1083 and $960 \mathrm{~cm}^{-1}$ (Additional file 1: Fig. S4).

Gel residues (if present) would appear as high-absorbance intensity pixels (red) on the green background of the 2D IR maps. For Xanthan gum (Fig. 8), small red pixels were identified in all test sites analysed; however, similar features were present in the control unsoiled/ untreated samples. When comparing the maps from the control (bottom row, A and B in Fig. 8) and cleaned areas (top row, $C$ and $D$ in Fig. 8), the $1530-1650 \mathrm{~cm}^{-1}$ region appears similar in all the samples, suggesting that Xanthan gum residues were not detected using this technique.

For the Shin-Etsu KSG350z emulsion-cleaned areas shown in Fig. 9, the silicon-methyl stretching absorption appears as a negative band (in reflectance mode); thus, gel residues should appear as low-absorbance intensity pixels (blue) on the green background. In this case, no significant differences were detected between the unsoiled control and treated areas; hence, no residues were detected using this technique. It is noted that other studies [22, 23] using techniques with even lower detection limits, such 

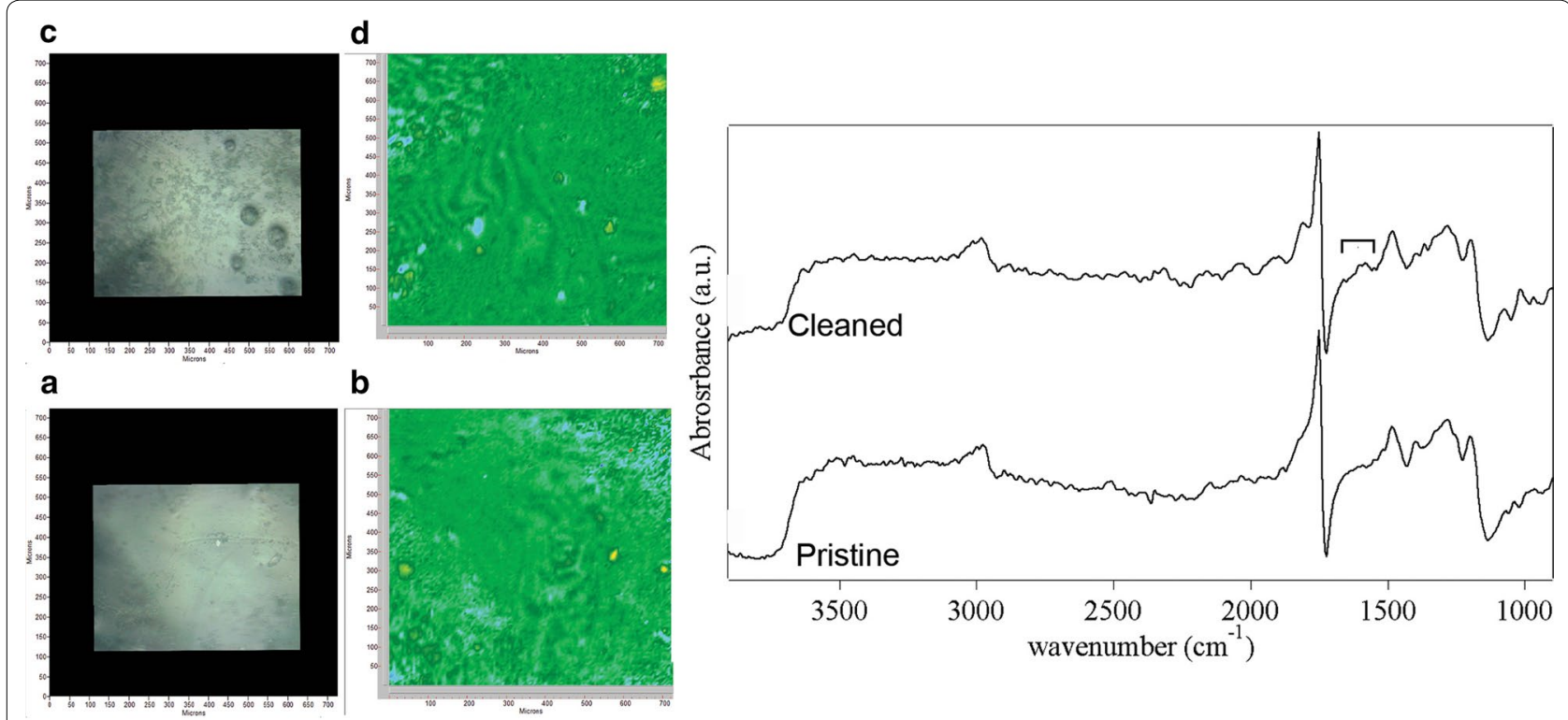

Fig. 8 FTIR-2D mapping for the axial deformation of $C=O$ of enols ( $\beta$-diketones); (1530-1650 cm ${ }^{-1}$ ) of Xanthan gum, for pristine area (i.e. unsoiled and untreated area) (bottom row, $\mathbf{a}$ and $\mathbf{b}$ ), and area cleaned with Xanthan gum (top row, $\mathbf{c}$ and $\mathbf{d}$ ). The corresponding FTIR spectra are shown in the right panel; each spectrum relates to a single pixel $\left(5 \times 5 \mu \mathrm{m}^{2}\right)$ of the corresponding 2D imaging map. Image $\odot$ Tate and CSGI

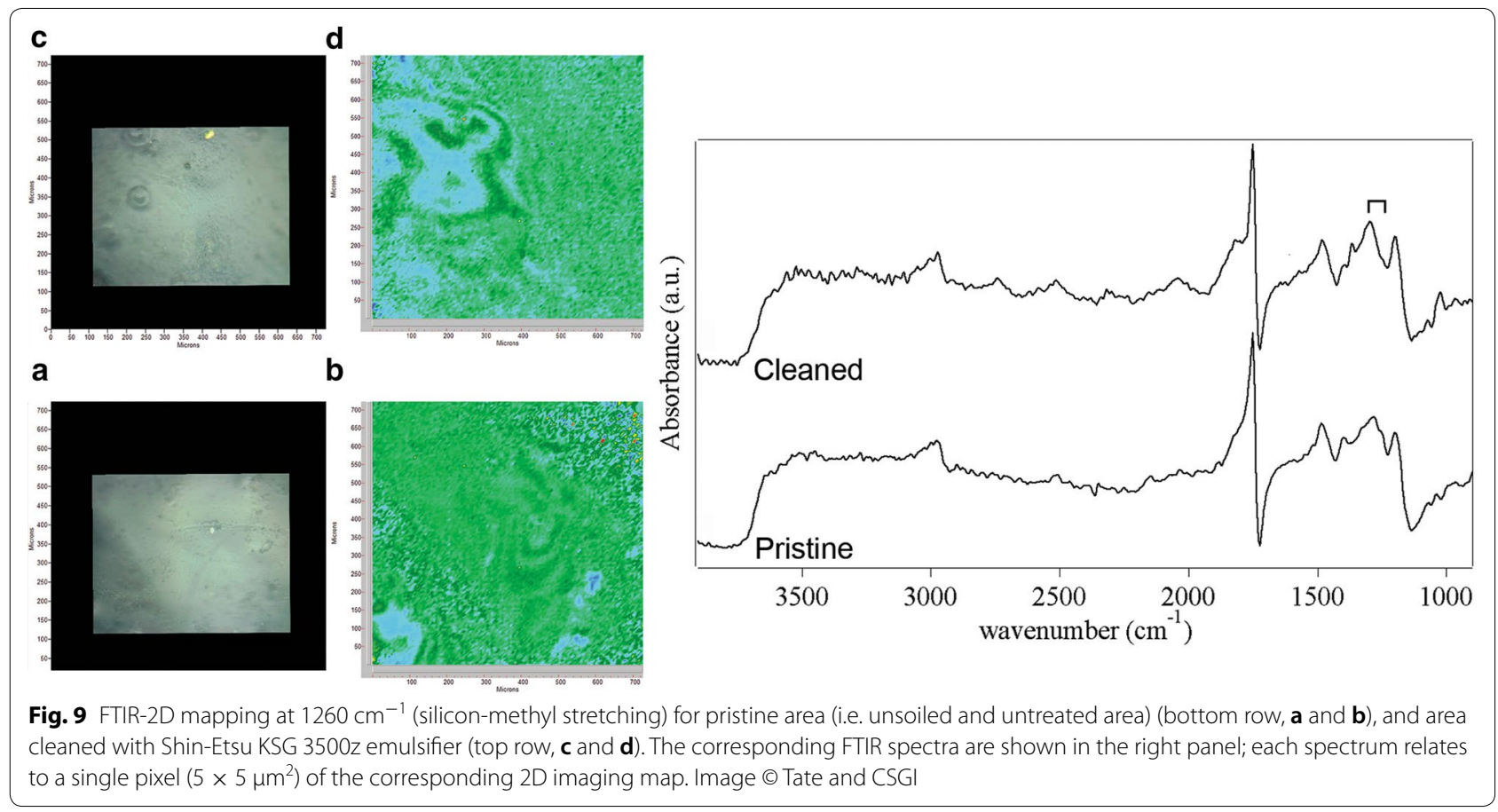

as XPS, have identified silicone emulsifier residues on paint films after clearance. ${ }^{17}$

\footnotetext{
${ }^{17}$ It is noted that the clearance procedure employed was performed repeatedly to minimise any residues on the highly textured mock-up samples.
}

The PVP absorption of the PVA/PVP based TT14 thin gel is an upward band in reflectance mode [40]; therefore, yellow-red pixels were expected where residues were present. As seen in Fig. 10, small blue areas are present in all treated locations; however, similar features are also 

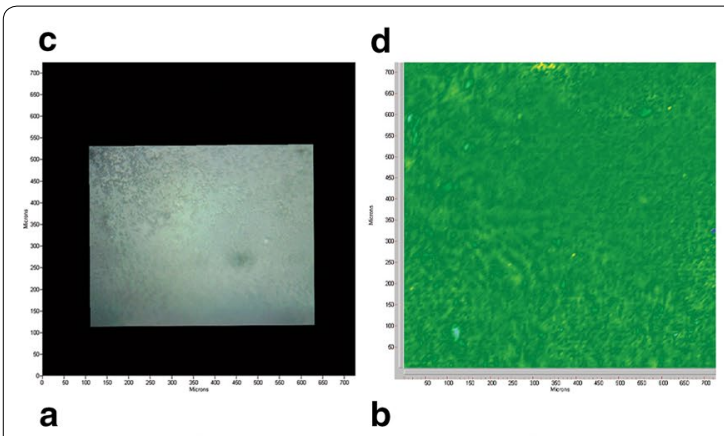

b
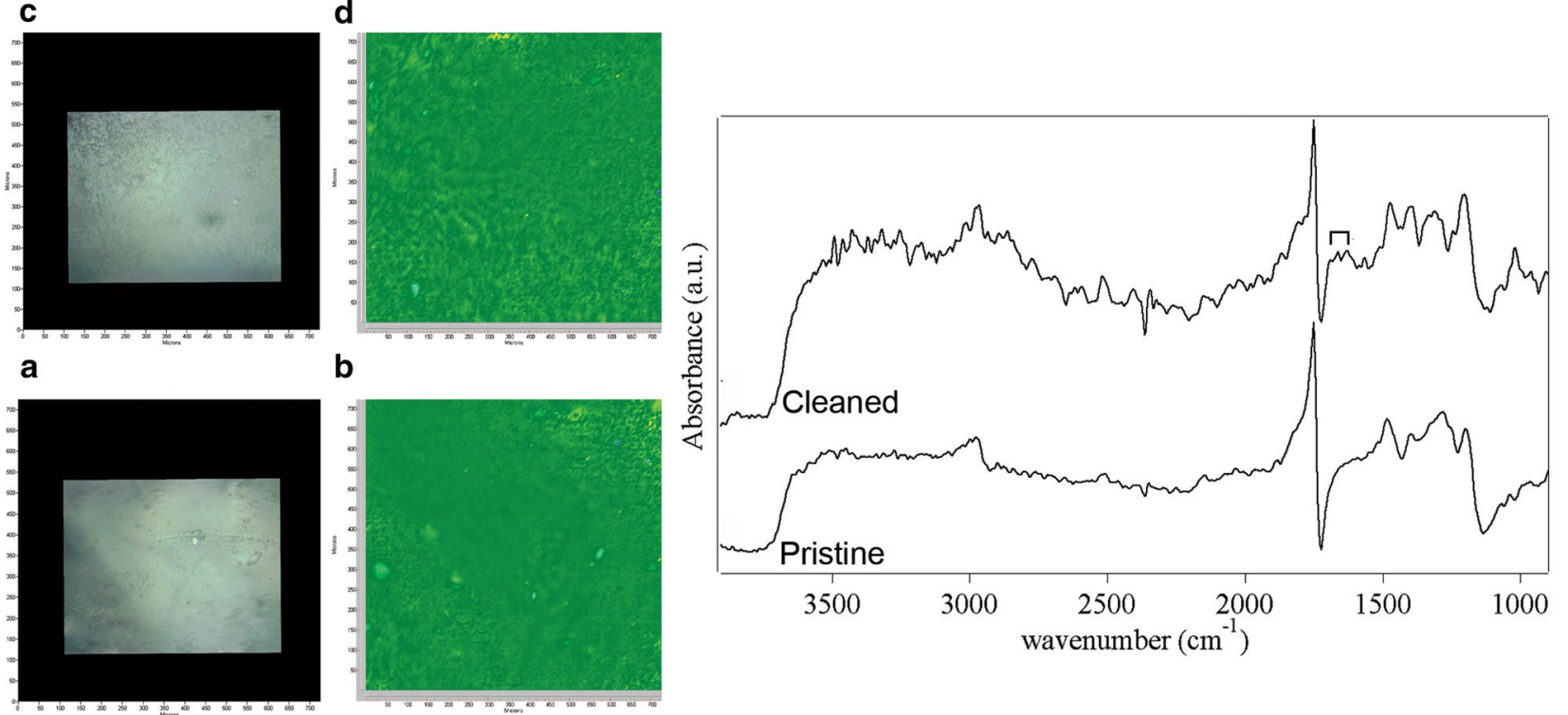

Fig. 10 FTIR-2D mapping at $1670 \mathrm{~cm}^{-1}(\mathrm{C}=\mathrm{O}$ stretching of PVP) for pristine area (i.e. unsoiled and untreated area) (bottom row, $\mathbf{a}$ and $\mathbf{b}$ ), and area cleaned with the PVA/PVP based TT14 thin gel (top row, $\mathbf{c}$ and $\mathbf{d}$ ). The corresponding FTIR spectra are shown in the right panel; each spectrum relates to a single pixel $\left(5 \times 5 \mu^{2}\right)$ of the corresponding $2 \mathrm{D}$ imaging map. Image $\odot$ Tate and CSGI

present in the control samples. By examining the spectra associated with these features, they could be assigned to the presence of the gelatine from the artificial soil, as indicated by the Amide I and Amide II absorption bands; hence in this case it also appears that no gel residues were detected using this technique.

Regarding the assessment of cleaning efficacy, this was measured through mapping the negative (downward) kaolin band present in the artificial soil, where azureblue pixels could be seen in maps of the soiled mock-ups, while green pixels were mostly observed in pristine references. Cleaning with all the three systems (Xanthan gum, Shin-Etsu KSG350z and TT14 thin gel), followed by appropriate clearance procedures, produced comparably effective levels of soil removal from the mock-ups, as shown in Additional file 1: Fig. S4.

\section{Results and discussion-part 2: sculpture treatment \\ Optimising the cleaning strategy-Addendum Papier mâché section}

None of the containing systems proved useful for cleaning the papier mâché section and, among the cosmetic sponges tested (Tables 2 and 3), only the NYX Pro Beauty Wedges and the SENSAI sponge cloth were further evaluated on the sculpture in combination with the most promising cleaning fluid selected from initial trials, i.e. an aqueous solution of ECOSURF ${ }^{\mathrm{TM}}$ EH-6/TAC (Additional file 1: Table S3) at concentrations varying from 0.5 to $1 \%$ w/w. Application using cosmetic sponges did not induce any undesirable changes to the surface and both sponges performed well, offering a homogeneous soiling removal, where the deeper areas of these highly textured surfaces were also successfully cleaned, as shown in Fig. 11 (top row). The cleaning efficacy was further improved using higher concentrations (i.e. $1 \% \mathrm{w} / \mathrm{w}$.) of both the surfactant and chelator as shown in Fig. 11 (top row). However, at these concentrations, foaming was noted at the surface; hence, the surfactant concentration was reduced to $0.75 \% \mathrm{w} / \mathrm{w}$.

\section{Rope section}

The systems taken forward for evaluation on the sculpture ropes included the blend of ECOSURF ${ }^{\mathrm{TM}}$ EH-6/TAC ( $1 \%$ and $2 \% \mathrm{w} / \mathrm{w}$. respectively), used with Xanthan gum and Shin-Etsu KSG350z as an emulsion, the Nanorestore Gel $^{\circledR}$ Peggy 6 (applied through clamping in the plumbing tube) and the optimal TT14 thin gel. Of these, the Shin-Etsu KSG350z emulsion proved to be the least suitable, primarily due to difficulties associated with satisfactory clearance from the textured rope surfaces, where residues were visible under magnification even after repeated clearance steps. The Xanthan gum cleaning efficacy results were comparable to the TT14 thin gel; however, the clearance procedure was time-consuming and resulted in slight disturbances to the rope surfaces, fibres and acrylic dispersion coating when viewed under magnification. 
ECOSURFm EH6 - TAC $[0.75 \%$ w/w]
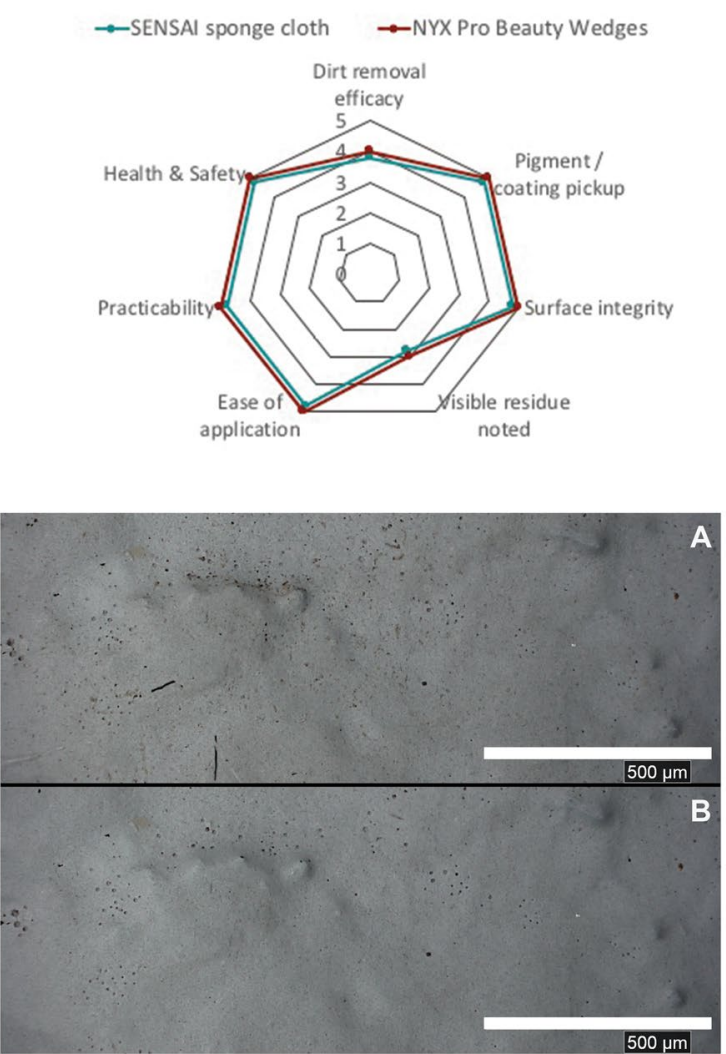

ECOSURF ${ }^{m}$ EH $6-$ TAC $[1 \%$ w/w]
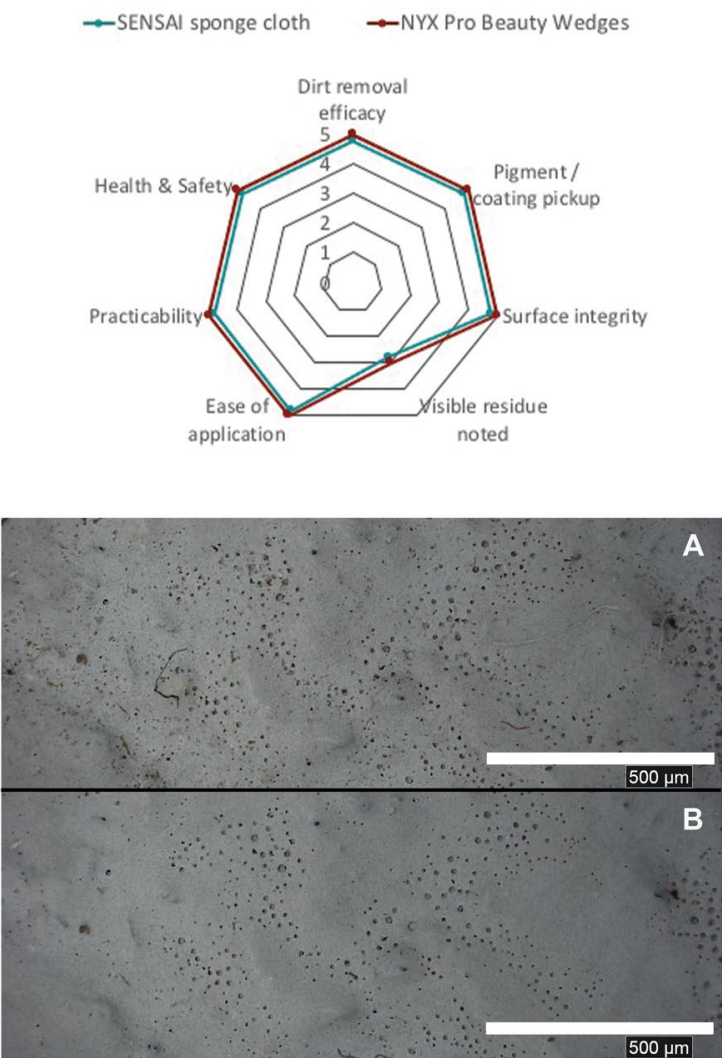

Fig. 11 Top row: Star diagrams summarising the performance of the aqueous cleaning solution at two different concentrations, tested and applied using NYX Pro Beauty Wedges and SENSAI sponge cloth. There were no differences between the two selected sponges with regards to the general cleaning performance. Bottom row: High-resolution (HIROX) images for before (a) and after (b cleaning tests performed on the top papier mâché section of the sculpture with SENSAl sponge cloth (left) and NYX Pro Beauty Wedges (right), loaded with a blend of ECOSURF ${ }^{\mathrm{TM}}$ EH-6/TAC (both at $1 \%$ w/w.). Clearance was performed with adjusted water 6:6. The scale bar is $500 \mu \mathrm{m}$. Photo $\odot$ Tate 2018

To optimise the Nanorestore Gel ${ }^{\circledR}$ Peggy 6 system, one test was performed using a ECOSURF ${ }^{\mathrm{TM}} \mathrm{EH}-6 / \mathrm{TAC}$ loaded gel with added 5\% w/w. propan-2-ol. ${ }^{18}$ For the Nanorestore Gel ${ }^{\circledR}$ Peggy and TT options, initial tests were performed using an exposure time of 2-min which was then increased at 2-min intervals until satisfactory results were achieved, up to a maximum of $10 \mathrm{~min}$ (Fig. 12). Satisfactory cleaning efficacy was obtained after 10-min exposures using the Nanorestore $\mathrm{Gel}^{\circledR}$ Peggy 6 option clamped as described earlier. ${ }^{19}$ After removal, no free liquid was noted on the rope surface, and no swelling or disturbance of the coating/paint layers/rope was observed. ${ }^{20}$ As expected, the addition of

\footnotetext{
18 The Nanorestore Gel ${ }^{\circledR}$ Peggy series is compatible with some polar solvents, up to $50 \%$ w/w. See http://www.csgi.unifi.it/products/peggy.html (accessed 12.12.19). The addition of propan-2-ol would have caused the slight swelling of the acrylic emulsion coating, which helped facilitate the removal of the more embedded soiling.
}

propan-2-ol helped to enhance cleaning efficacy and facilitated the reduction of the exposure time to 8-min. After further tests, the TT14 thin gel loaded with the blend of ECOSURF ${ }^{\mathrm{TM}}$ EH-6/TAC proved most effective at soiling removal and offered the most suitable application to the ropes. As was noted for the mock-up ropes, this gel adhered optimally to the substrate and to itself, which enabled even soil removal after a 6-min exposure. In this case, the gel-face in contact with the rope appeared homogeneously discoloured, confirming an even cleaning action had been achieved across the treatment area.

\footnotetext{
$\overline{{ }^{19} \text { Clearance was performed with a Nanorestore Gel }{ }^{\circledR} \text { Peggy hydrogel clamped }}$ in same plumbing tube, for the same exposure time.

${ }^{20}$ Measurements of the rope diameter were taken with a micrometre before, straight after and after one day post-cleaning for all cleaning materials assessed; no significant differences were observed.
} 


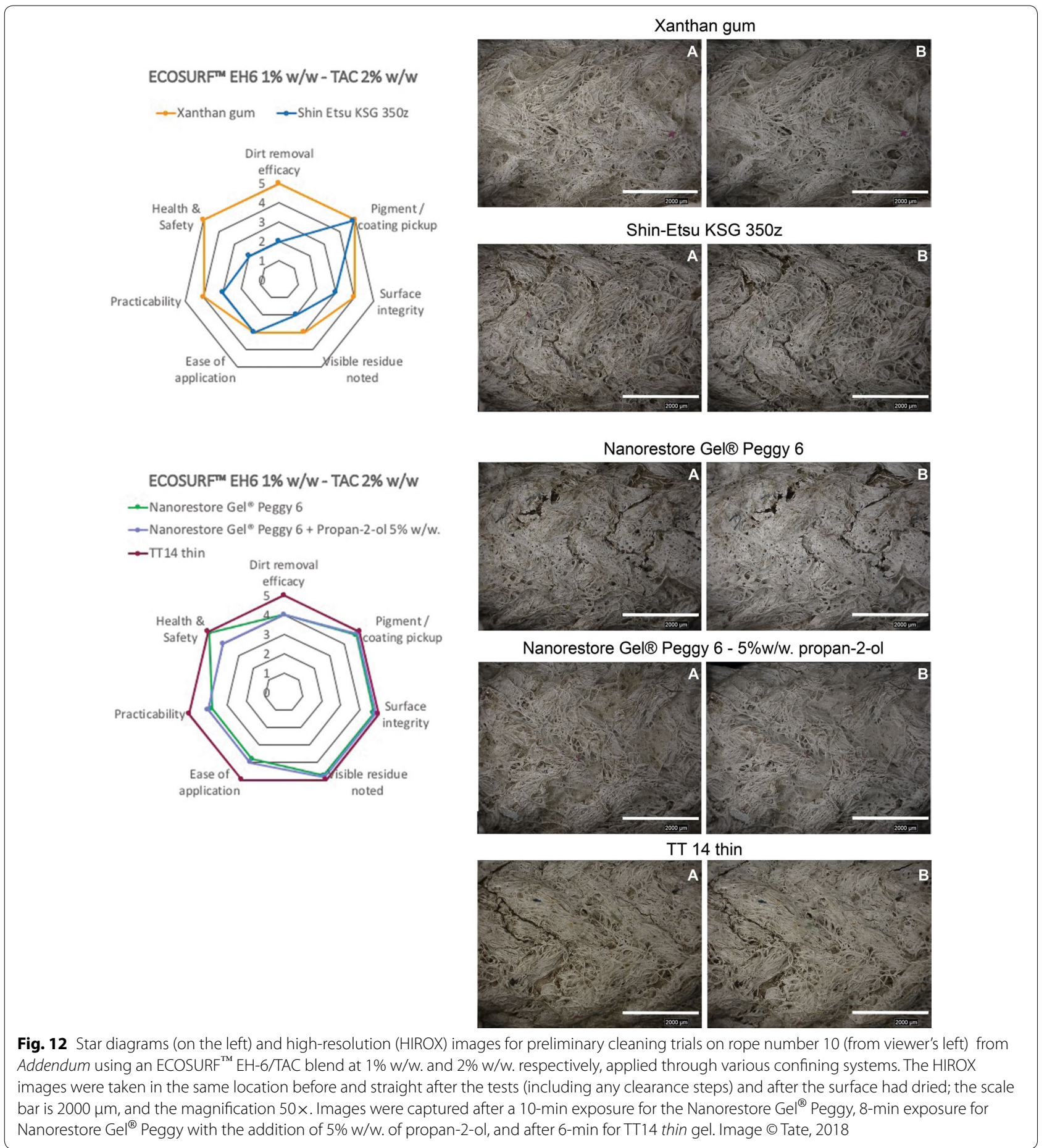

Based on these considerations, the TT14 thin (PVA/ PVP) gel was chosen as the most suitable system to take forward to Addendum, due to its high cleaning efficacy (Fig. 13) and minimal mechanical action. Additional trials were performed by varying the TAC concentrations within the aqueous blend up to a maximum of $5 \% \mathrm{w} / \mathrm{w}$., as well as through testing the TT14 thin gel loaded with other cleaning solutions derived from free-liquid trials on the sculpture (Additional file 1: Table S3). However, the blend of ECOSURF ${ }^{\mathrm{TM}} \mathrm{EH}-6 / \mathrm{TAC}$ (at $1 \%$ and $2 \%$ w/w. respectively) with added propan-2-ol (at 5\% w/w.) 

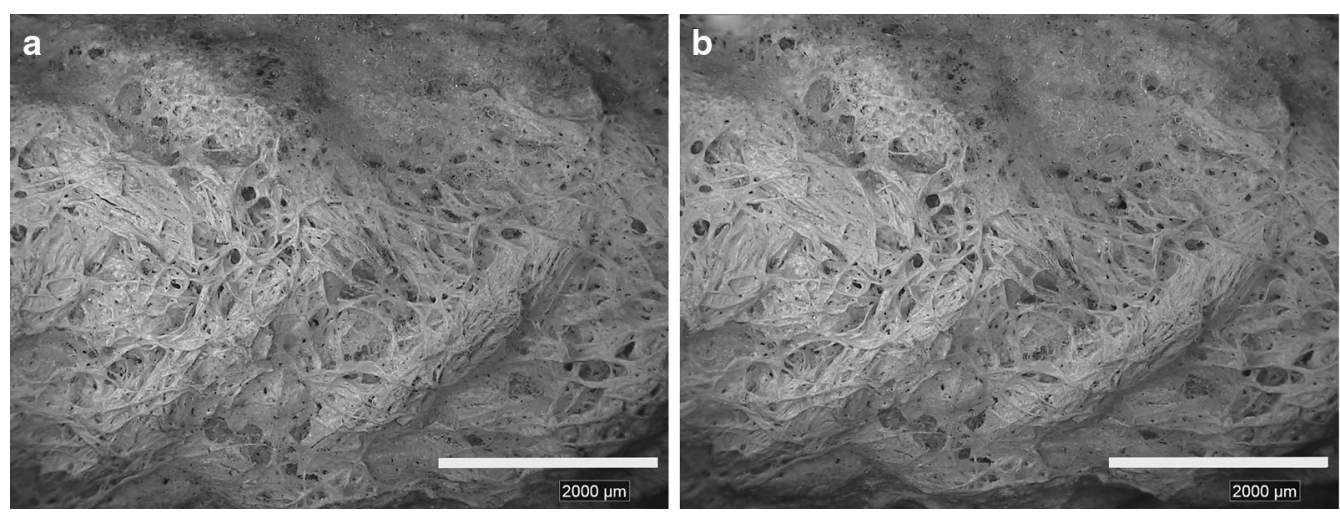

Fig. 13 High-resolution (HIROX) images of an area of rope number 10 from Addendum before (a) and after (b) cleaning test with TT14 thin gel loaded with an aqueous solution containing ECOSURF ${ }^{\mathrm{TM}} \mathrm{EH}-6$ and TAC ( $1 \%$ and $2 \% \mathrm{w} / \mathrm{w}$. respectively) for a 6 -min exposure (followed by the appropriate clearance step with a TT14 thin hydrogel). There was no evidence of any alteration to the rope surface, which also appeared less discoloured. The soil embedded in the uppermost coating layer was also reduced. The scale bar is $2000 \mu \mathrm{m}$, magnification 50x. Photo @ Tate 2018

consistently offered the highest cleaning efficacy, with no undesirable changes observed.

\section{Final protocol for Addendum}

Once optimised, the soiling removal phase of the conservation treatment took approximately 4 months to complete and involved the use of $60 \times$ TT14 thin gels. The treatment commenced at the lowest part of the ropes, due to the need to closely monitor the changing tonal balance between the ropes and the upper papier mâché section as the cleaning treatment progressed.

The treatment was performed as follows:

1. The ropes were cleaned using the TT14 thin gel, loaded with an aqueous solution with added $5 \% \mathrm{w} / \mathrm{w}$. propan-2-ol, TAC (2\% w/w.) and ECOSURF ${ }^{\mathrm{TM}}$ EH- 6 at $0.75 \% \mathrm{w} / \mathrm{w}$ for up to 6 -min exposures. Clearance was performed using TT14 thin hydrogels (i.e. TT14 thin gel loaded with deionised water) applied for the same time.

2. The gels, received in $15 \times 10 \mathrm{~cm}$ sheets ready for use, were cut into strips of approximately $5-10 \mathrm{~mm}$ width and prepared for application by soaking overnight in the cleaning solution (cleaning gels) or deionised water (clearance gels).

3. Before application, while wearing gloves, one strip was removed from the cleaning gel bath and firmly dried by blotting onto layers of Evolon tissue and blotting paper, until the gel developed a sticky consistency (NB: the drier the gel, the better the adhesion to the substrate).

4. The gels were applied to the bottom of each rope, by wrapping each strip in a spiral (Fig. 14a) and left for the allotted application time. A cotton tie (Fig. 14b) was used on either end of the gel to mark where the treated area ended. During application, the gel was lightly pressed using gloved fingers to ensure optimal contact between the gel and the rope.

5. After exposure, the cleaning gel was unwrapped and placed aside for re-use. Upon removal, the side of the gel in contact with the rope surface mirrored the texture of the rope and appeared discoloured, confirming that soiling had been absorbed onto the gel structure (Fig. 14d).

6. The clearance step was performed immediately using the TT14 thin hydrogel using the same procedure. The next set of cleaning and clearance gels were applied following the same steps, while slightly overlapping onto the previously cleaned area to ensure even cleaning.

7. Once each gel had been used on both sides, it was placed in a deionised water bath for cleaning overnight (with other used gels) and reused approximately three times.

8. In some particularly soiled areas (i.e. the rope ends in contact with the floor), the treatment was repeated to remove additional soiling and/or to even out tonality.

As described earlier, the papier mâché section was optimally cleaned (as evident in Fig. 15) using an aqueous solution containing TAC $(1 \% \mathrm{w} / \mathrm{w}$.) and the non-ionic surfactant ECOSURF ${ }^{\mathrm{TM}}$ EH-6 (0.75\% w/w.). This blend was applied using two methods, the SENSAI sponge cloth and NYX Pro Beauty Wedges, as follows:

9. The SENSAI sponge cloth was loaded by immersion, and excess liquid removed through squeezing. The sponge was then applied to the surface using a 

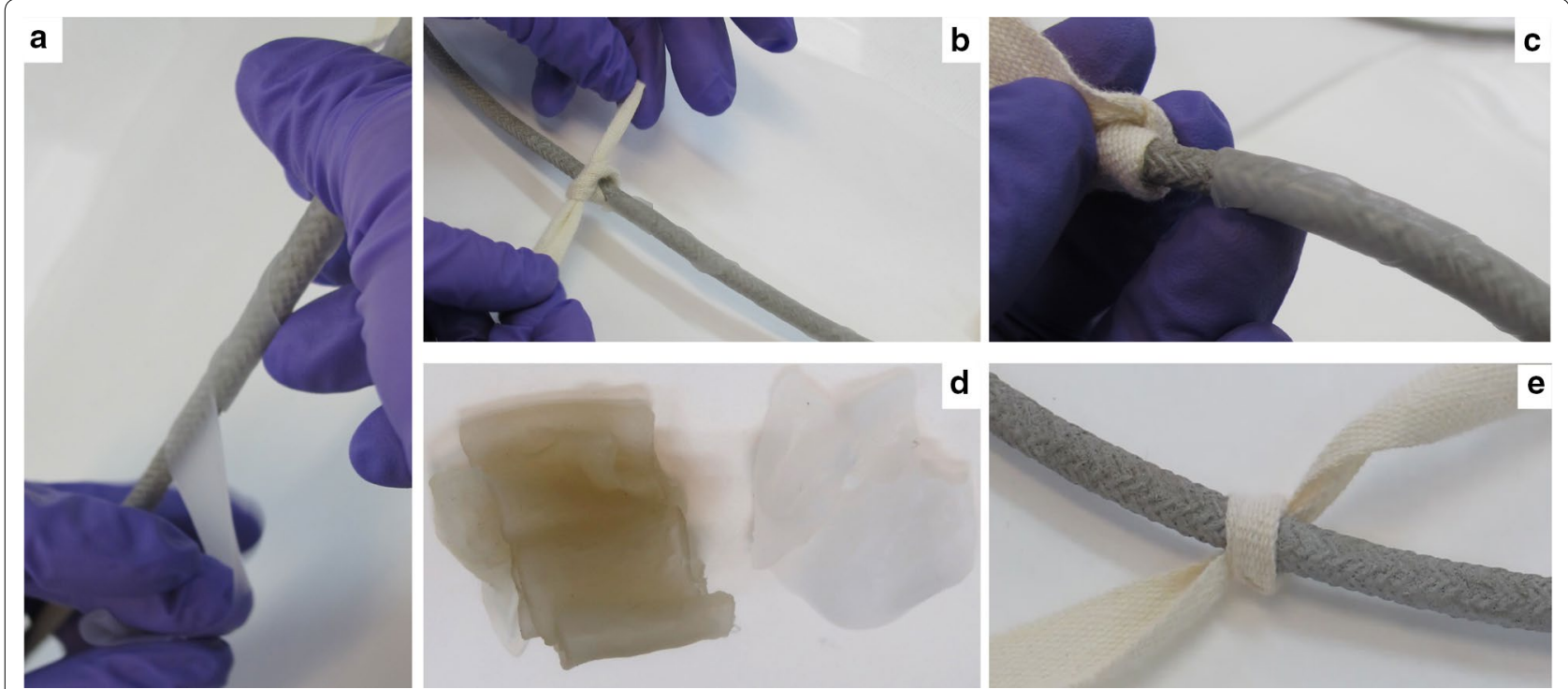

Fig. 14 Details of the cleaning procedure used on Addendum's ropes. The gel was wrapped around the rope in a spiral (a), and cotton ties were used to mark cleaning site (b). The gel is very thin and transparent, enabling visual access to the rope underneath, and sticks perfectly to itself and the substrate (c). Upon removal, the side of the gel in contact with the rope surface appeared discoloured compared to the clearance gel, confirming that soiling had been absorbed into the gel structure (d). Cleaned area (e, to the right of the cotton tie) and an area yet to be cleaned (e, to the left of the cotton tie). Photos $\odot$ Tate 2018

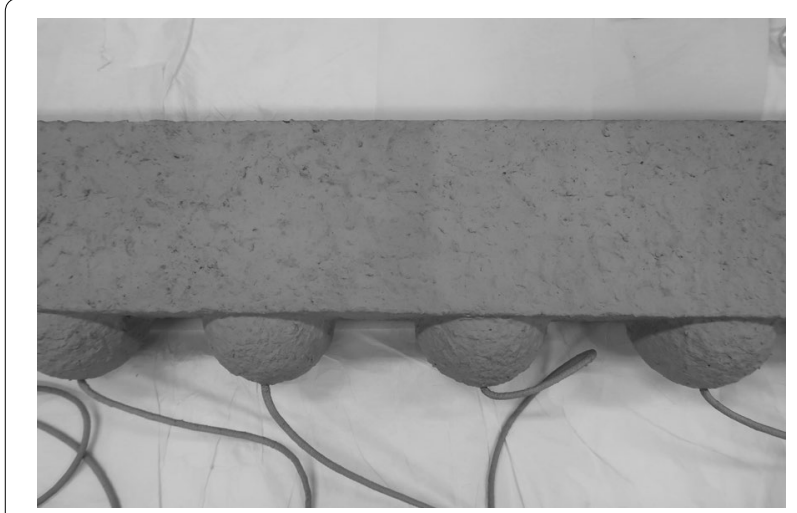

Fig. 15 Addendum papier mâché section halfway through cleaning, where the difference between the left (yet to be cleaned) and right (cleaned) areas is evident. Photo Tate, 2018. @ The estate of Eva Hesse, courtesy Hauser \& Wirth, Zürich

gentle circular motion and a second cleaning step was performed using the NYX sponge which was loaded, blotted and applied in the same way. The SENSAI sponge was chosen for the initial cleaning step due to its thinness (approximately 1-3 mm), which offered an informative connection to the sculpture surface. The NYX sponge was applied for a general overall clean and accessed areas where the SENSAI sponge had not removed all the soil.
10. For accuracy, the areas around the holes of the 17 hemispheres (from which the ropes originate), were cleaned using a small hand-rolled cotton swab.

11. Clearance was performed across the papier mâché section using a NYX sponge loaded with adjusted water 6:6, which was blotted and applied as described above.

\section{Retouching and post-cleaning assessment}

An old, discoloured retouching dating back to 1979, located on the 6th hemisphere from the left (viewer's left), was also removed during the cleaning treatment and retouched using titanium white, Mars black pigments, with added kaolin (Kremer Pigmente, Germany) mixed into Lascaux Water Resoluble Medium (Amazon, UK). Areas of paint loss along the bottom edge of the upper section were filled using Flügger (Conservation Resources Ltd, UK) ${ }^{21}$ and retouched using the same mixture. On the top edge, areas of loss were toned in using the same mixture and small areas of flaking papier mâché (along the top edge) were consolidated with Plextol B500 (Conservation Resources Ltd, UK).

\footnotetext{
${ }^{21}$ https://conservation-resources.co.uk (accessed 18.12.19).
} 


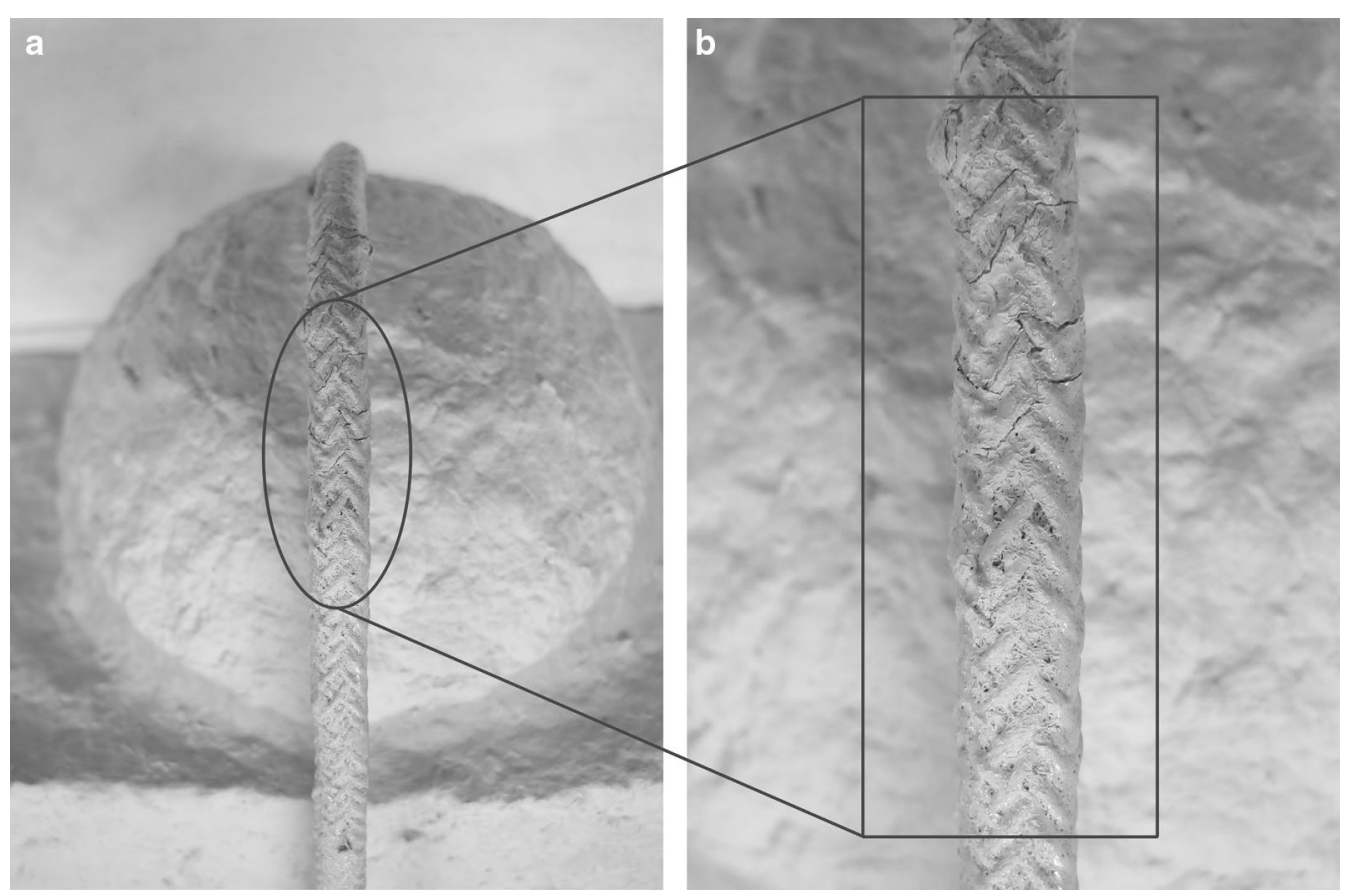

Fig. 16 The removal of the yellowed soiling from the ropes enabled a better colour uniformity between the papier mâché and the ropes (a), particularly in areas where the uppermost p(nBA-MMA) coating was thin or not present (b). Photos Tate, $2018 \odot$ The estate of Eva Hesse, courtesy Hauser \& Wirth, Zürich

The initial cleaning tests carried out on rope number 9 (viewer's left) had resulted in some slightly cleaner areas, which were toned back using Mars Black pigment mixed into Lascaux Water Resoluble Medium.

Due to the inherent texture of this work, assessment techniques better suited to flat surfaces (such as colour and gloss measurement) could not be used; however, microscopy was extensively used to evaluate the trials and the post-treatment surface of the sculpture. The post-treatment examinations revealed that no change had occurred other than the removal of the yellowed, particulate soiling, which, to a great extent, had recovered the cooler grey colour and more uniform tone across the 17 ropes (Fig. 16). The changes in tone resulting from the treatment were monitored carefully and discussed on a regular basis with a wide group of stakeholders. Ultimately, the cleaning treatment was deemed successful in restoring a higher degree of uniformity between the papier mâché section and the 17 ropes, which was particularly evident when the work was returned to display (Fig. 17). ${ }^{22}$

\footnotetext{
${ }^{22}$ The return of Addendum to display was marked at Tate Modern in 2018; and while the display configuration remains the same, the work was hung away from air-conditioning vents and areas more prone to the accumula-
}

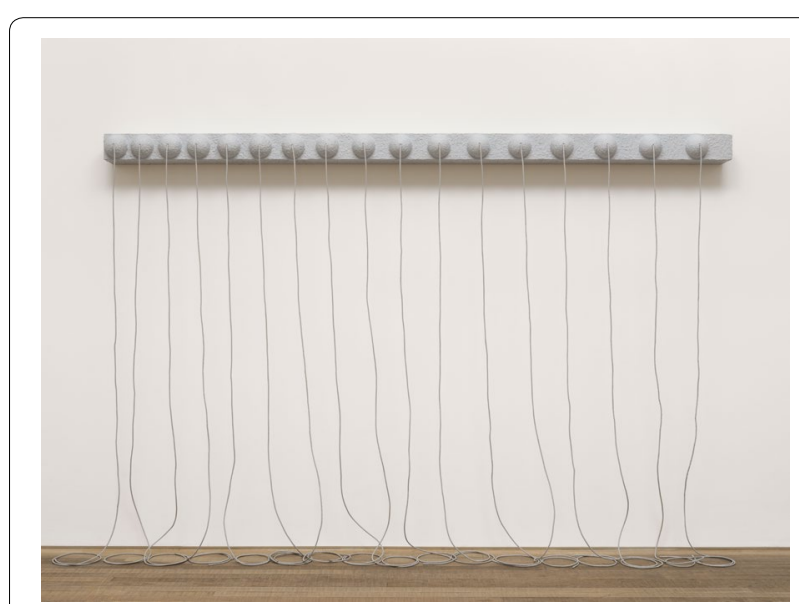

Fig. 17 Addendum after cleaning and on display at Tate Modern. Photo Tate, July 2018 @ The estate of Eva Hesse, courtesy Hauser \& Wirth, Zürich

tion of dust. It is expected that this work will need to be cleaned again and should be assessed every 1-2 years for the unwanted accumulation of soiling, depending on the frequency of display. 


\section{Conclusions}

This paper presents rigorous, practice-based research which supported, facilitated and guided the first surface cleaning treatment of Eva Hesse's sculpture Addendum (Fig. 17). The methodology employed produced new insights into Hesse's material practice and aesthetic, and facilitated a reflective, iterative evaluation of a range of established and novel cleaning systems, several of which were also assessed for possible residues. The treatment optimisation research was tailored to each section of the sculpture, in each case via exploring which solvents would form the base of wet-cleaning systems, followed by optimising the most appropriate solvents through the modification of $\mathrm{pH}$, conductivity, the addition of surfactants and chelators, and controlling their application using spreadable, mouldable, rigid and novel gels as well as silicone emulsifiers. Each evaluation step built on previous results, having been designed to closely follow the conservation treatment decision-making process.

As systems were trialed and evaluated on mock-ups and as discreet tests on the sculpture, several options were discarded due to unacceptable levels of risk and/or poor contact and/or cleaning efficacy.

As the evaluations progressed, optimised aqueous solutions loaded into Xanthan gum, Shin-Etsu KSG 350z silicone emulsifier and the Nanorestore ${ }^{\circledR}$ Peggy gel series proved most promising. Trials with these materials on the rope mock-ups prompted further modifications to the Nanorestore Gel ${ }^{\circledR}$ Peggy 5 synthesis procedure to create gels with enhanced tackiness and self-adhesion, known as the TT series. ${ }^{23}$ From this, the TT14 thin gel could be successfully wrapped around the ropes without the need for clamping or other invasive procedures, which, when combined with an optimised aqueous cleaning solution, enabled the successful, low-risk removal of embedded soiling from the 17 ropes. When combined with the removal of soiling from the papier mâché section using optimised aqueous solutions applied with sponges and cloths, the cleaning treatment was highly successful in recovering the uniformity between the papier mâché section and the 17 ropes, returning the work to a more authentic state.

It is important to note however, that even as specific materials were deemed unsuitable for this case study, each of the materials evaluated presented advantages and disadvantages, which may render them better suited for use on other works of art. Equally, the cleaning system residue study presented was carried out on

\footnotetext{
${ }^{23}$ The TT 14 thin version of the Nanorestore ${ }^{\circledR}$ Peggy 5 hydrogel has been thus far produced only at the lab-scale, owing to the longer, more extensive synthesis procedure required.
}

a narrowed selection of gels and emulsifiers which warrant further exploration, particularly when used at higher concentrations than those evaluated here, and where other types of cleaning agents (e.g. chelators, surfactants) have been used.

\section{Supplementary information}

Supplementary information accompanies this paper at https://doi. org/10.1186/s40494-020-00378-z.

Additional file 1: Fig. S1. The papier mâché board, hemisphere and rope mock-up samples with Addendum in the background (A). Papier mâché board control (B) and with artificial soiled applied (C). Photos, Tate @ The estate of Eva Hesse, courtesy Hauser \& Wirth, Zürich. Fig. S2. Application method of a strip of Nanorestore Gel ${ }^{\circledR}$ Peggy 6 wrapped in spiral around a rope mock-up (A), with various strategies to ensure constant contact with the substrate, including cling film wrapped both at the end of the gel as well as around the entire gel strip (B); a small clamp used to fix and keep the gel ends in position (C); a small piece of plumbing pipe, cut in half and lined with Plastazote foam and Melinex (D) used to keep the gel in place during treatment (E). Photos @ Tate. Fig. S3. Star diagram for cleaning tests performed on the rope mock-ups, with an optimised aqueous fluid (i.e. ECOSURF ${ }^{\mathrm{TM}} \mathrm{EH}-6 / \mathrm{TAC}$ blend at $1 \% \mathrm{w} / \mathrm{w}$. and $2 \% \mathrm{w} / \mathrm{w}$. respectively) loaded into 4 containing systems: Xanthan gum; Shin-Etsu KSG350z; Nanorestore Gel ${ }^{\circledR}$ Peggy 6; TT14 thin. Image ( Tate. Fig. S4. FTIR 2D mapping of the mock up sample, showing a control area with artificial soil applied (A, B), a control unsoiled area (C, D), and areas cleaned using an ECOSURF ${ }^{\mathrm{TM}} \mathrm{EH}-6 / \mathrm{TAC}$ blend at $1 \% \mathrm{w} / \mathrm{W}$. and $2 \% \mathrm{~W} / \mathrm{W}$. W/W. respectively, applied through various confining systems, i.e. Xanthan gum (E, F), Shin-Etsu KSG 3500z $(G, H)$ and TT14 thin gel $(I, J)$. For each area, the image besides the visible map shows the corresponding 2D FTIR Imaging map, where the intensity of the band between 1083 and $960 \mathrm{~cm}^{-1}$ (assigned to the $\mathrm{Si}-\mathrm{O}$ stretching of kaolin) was imaged. The corresponding FTIR spectra are shown in the right panel; each spectrum relates to a single pixel $(5 \times$ $5 \mathrm{\mu m}^{2}$ ) of the corresponding 2D imaging map. All maps have dimensions of $700 \times 700 \mu^{2}$. Image $\odot$ Tate and CSGI. Table S1. List of criteria and description of star diagram rating scales used to evaluate free- fluid options on Addendum. Table S2. List of criteria and description of star diagram rating scales used to evaluate the gels and emulsifiers on both mock-up samples and Addendum. Table S3. Key observations after trials of non-confined cleaning solutions (10-swab roll applications) on both papier mâché and rope mock-ups and Addendum. Clearance strategies are reported in Table 2. N.B: For all tests performed on Addendum's rope section, the surface appeared brittle and sensitive to mechanical action. Table S4. Key observations for tests on the mock-up samples with the TT series, modified from Nanorestore Gel ${ }^{\circledR}$ Peggy 5 (PVA/PVP-based hydrogels). These gels were specifically synthesized for use on the rope replica, with one option (TT16 thick) designed for the highly-textured papier mâché section.

\section{Abbreviations}

PVA: Polyvinyl alcohol; PVP: Polyvinyl pyrrolidone; PVAc: Polyvinyl acetate; p(EA-MMA): Poly(ethyl acrylate-methyl methacrylate); p(nBA-MMA): Poly(butyl acrylate-methyl methacrylate); EA: Ethyl acrylate; MMA: Methyl methacrylate; BA: Butyl acrylate; DEP: Diethyl phthalate; PW6: Pigment white 6; PBk7: Pigment black 7; UV: Ultraviolet; FTIR: Fourier Transform InfraRed spectroscopy; ATR: Attenuated Total Reflectance; 2D: Two dimensional; SEM: Scanning Electron Microscopy; EDX: Energy dispersive X-ray analysis; PyGCMS: Pyrolysis gas chromatography mass spectrometry; AMU: Atomic mass units; DI: Deionised water; Adjusted water 6: 6 : adjusted water at $\mathrm{pH} 6$ and conductivity $6 \mathrm{mS} / \mathrm{cm}$; MES: 2-(N-morpholino)ethanesulfonic acid; D5: Decamethylcyclopentasiloxane; TAC:Triammonium citrate; EDTA: Ethylenediaminetetraacetic acid; DPTA: Diethylenetriaminepentaacetic acid; NaDOSS: Anionic surfactant dioctyl sulfosuccinate, sodium salt; NANORESTART: NANO materials for the RESToration of ART; PPE: Personal protective equipment. 


\begin{abstract}
Acknowledgements
The authors would like to acknowledge and thank the following people and institutions: all NANORESTART partners; Tate Conservation and Research Departments - in particular Judith Lee, Joyce Townsend, Luigi Galimberti, Pip Laurenson, Deborah Potter and Deborah Cane; Tate Curatorial Departmentin particular Matthew Gale, Gregor Muir and Frances Morris; Tate Photography Department; Nelly von Anderkas, UK; Bryony Fer, UK; Barry Rosen and the Estate of Eva Hesse, US; Chris Stavroudis, Private practice, US; Tom Learner, Getty Conservation Institute, US; Melinda H. Keefe, The Dow Chemical Company, US; Stuart Croll, formerly NDSU, US; Mark Golden, Golden Artist Colors, US; and finally, the peer reviewers and Richard Brereton.
\end{abstract}

\section{Authors' contributions}

BAO designed, led and managed Tate's contribution to the NANORESTART project. BAO, AB and TM informed the cleaning tests, analysis and methodologies used. TM carried out research into the appearance and art historical context of the sculpture; determined the specific and broader aims of the cleaning treatment; carried out the cleaning tests and the full conservation treatment, with support from $A B$ and $B A O$. $A B$ and $B A O$ performed the sculpture materials analysis and data interpretation of the cleaning trial results, with LVA (formerly Tate) and DC (CSGI) supporting the artwork analysis and FPAFTIR analysis and interpretation respectively. NB synthesised the Nanorestore Gel ${ }^{\circledR}$ Peggy and TT series used for the research phase and the final conservation treatment. $A B$ produced the first draft of the manuscript and contributed to several edits; $\mathrm{BAO}$ and TM contributed text to the manuscript, and BAO fully edited the manuscript prior to submission. All authors read and approved the final manuscript.

\section{Authors' information}

Bronwyn Ormsby is Principal Conservation Scientist at Tate (London, U.K.) and designed, led and managed Tate's contribution to the NANORESTART project (2015-2018) with the support of Tate's Research and Conservation Departments. The wider NANORESTART project was led by Professor Piero Baglioni (University of Florence \& CSGI, Italy). David Chelazzi [DC], researcher at CSGI, was a coordinator of the project. Angelica Bartoletti [AB] was the Tate NANORESTART Post-doctoral Conservation Science researcher between 2017 and 2019; Lora V. Angelova [LVA] was the Tate NANORESTART Post-doctoral Conservation Science researcher in 2016-2017. Tamar Maor [TM] was the Tate NANORESTART Conservator for this case study (2017-2018).

\section{Funding}

This research was conducted within the framework of the NANORESTART project, which received funding from the European Union's Horizon 2020 research and innovation programme under Grant Agreement No 646063. The purchase of the HIROX KH-8700 digital microscope and the final conservation treatment of Addendum were conjunctively supported by the NANORESTART project funding and Tate.

\section{Availability of data and materials}

All the data are available within the manuscript.

\section{Competing interests}

The authors declare that they have no competing interests.

\footnotetext{
Author details

${ }^{1}$ Conservation Department, Tate, Millbank, London SW1P 4RG, UK. ${ }^{2}$ Present Address: Department of Conservation and Restoration and Research Unit LAQV-REQUIMTE, NOVA School of Sciences and Technology (FCT NOVA), 2829-516 Monte da Caparica, Portugal. ${ }^{3}$ Present Address: TM Art Conservation, Houston, TX, USA. ${ }^{4}$ Department of Chemistry, University of Florence and CSGI, Via della Lastruccia 3, Sesto Fiorentino, 50019 Florence, Italy.

${ }^{5}$ Present Address: The National Archives, Kew, Richmond, Surrey, London TW9 4DU, UK.
}

Received: 19 December 2019 Accepted: 30 March 2020

Published online: 11 April 2020

\section{References}

1. Murray A, Contreras de Berenfeld C, Chang SYS, Jablonski E, Klein T, Riggs MC, Robertson EC, Tse WMA. The condition and cleaning of acrylic emulsion paintings. In: Vandiver PB, Goodway M, Mass JL, editors. Proceedings from Symposium II: materials issues in art and archaeology VI, Boston, 26-30 November 2001. Boston: Material Research Society; 2002. pp. 1-8.

2. Jablonski E, Learner T, Hayes J, Golden M. Conservation concerns for acrylic emulsion paints. Stud Conserv. 2003;48:3-12.

3. Learner T, Smithen P, Krueger J, Schilling M, editors. Modern paints uncovered: proceedings from the modern paints uncovered symposium, London (UK), 16-19 May 2006. Los Angeles: Getty Conservation Institute; 2007.

4. Chiantore O, Rava A, editors. Conserving contemporary art: issues, methods, materials, and research. Los Angeles: The Getty Conservation Institute; 2012.

5. Lippard LR. Eva Hesse. New York: New York University Press; 1976.

6. Tate conservation report file for Eva Hesse Addendum (T02394).

7. Ormsby B, Keefe M, Phenix A, Von Aderkas E, Learner T, Tucker C, Kozak C. Mineral spirits-based microemulsions: A novel cleaning system for painted surfaces. J Am Instit Conserv. 2016;55:12-31.

8. Ormsby B, Learner T, Schilling M, Druzik J, Khanjian H, Carson D, Foster G, Sloan $M$. The effects of surface cleaning on acrylic emulsion paintings. A preliminary investigation. In: Weyer C, editor. Oberflächenreinigung; Materialien und Methoden, VDR-Schriftenreihe 2: Proceedings from the conference Surface cleaning - material and methods, Düsseldorf, 29 September-4 October 2003. Stuttgart: Theiss Verlag. 2006. pp. 135-49.

9. Ormsby B, Learner T, Foster G, Druzik J, Schilling M. Wet-cleaning acrylic emulsion paint films: an evaluation of physical, chemical and optical changes. In: Learner T, Smithen P, Krueger J, Schilling M, editors. Modern paints uncovered: proceedings from the modern paints uncovered symposium, London (UK), 16-19 May 2006. Los Angeles: Getty Conservation Institute; 2007. pp. 189-200.

10. Ormsby $B$, Learner T. The effects of wet surface cleaning treatments on acrylic emulsion artists' paints - a review of recent scientific research. Stud Conserv. 2009;54:29-41.

11. Kampasakali, E, Ormsby B, Cosentino A, Miliani C, Learner T. A preliminary evaluation of the surfaces of acrylic emulsion paint films and the effects of wet-cleaning treatment by atomic force microscopy (AFM). Stud Conserv. 2011;56:216-30

12. Dillon CE, Lagalante AF, Wolbers RC. Acrylic emulsion paint films: the effect of solution $\mathrm{pH}$, conductivity, and ionic strength on film swelling and surfactant removal. Stud Conserv. 2014;59:52-62.

13. Lagalante AF, Wolbers RC. Particle-based silicone cleaning emulsions: studies in model paint systems for the cleaning of water-sensitive artworks. In: Angelova LV, Ormsby B, Townsend JH, Wolbers R, editors. Gels in the Conservation of Art. London: Archetype Publications Ltd; 2017. pp. 193-9.

14. Lagalante AF, Wolbers RC. The cleaning of acrylic paintings: new particle-based water-in-oil emulsifiers. In: Selva Bonino VE, editor. Dall'olio all'acrilico, dall'impressionismo all'arte contemporanea. Studi, ricerche, indagini scientifiche ed interventi conservativi: Proceedings from the VII Congresso Internazionale Colore e Conservazione (CESMAR7), Milan (Italy), 13-14 November 2015. Milan: il Prato Publishing; 2016. pp. 163-76.

15. Bartoletti A, Barker R, Chelazzi D, Bonelli N, Baglioni P, Lee J, Angelova LV, Ormsby BA. Reviving WHAAM! a comparative evaluation of cleaning systems for the conservation treatment of Roy Lichtenstein's iconic painting. Herit Sci 2020;8:9.

16. Maor T, Bartoletti A, Ormsby BA. Insight into Eva Hesse's working practice: a technical study of Addendum. Tate Papers, no 33, Spring 2020.

17. Whitmore PM, Colaluca VG. The natural and accelerated aging of an acrylic artists' medium. Stud Conserv. 1995;40:51-64.

18. Learner T, Chiantore $O$, Scalarone D. Ageing studies of acrylic emulsion paints. In: Vontobel R, editor. ICOM-CC 13th Triennial Meeting Preprints, Rio de Janeiro, 22-27 September 2002. London: James \& James; 2002. pp. 911-9. 
19. Smith GD. Ageing characteristics of a contemporary acrylic emulsion used in artists's paint. In: Learner T, Smithen P, Krueger J, Schilling M, editors. Modern paints uncovered: proceedings from the modern paints uncovered symposium, London (UK), 16-19 May 2006. Los Angeles: Getty Conservation Institute; 2007. pp. 236-46.

20. Ormsby BA, Soldano A, Keefe MH, Phenix A, Learner T. An empirical evaluation of a range of cleaning agents for removing dirt from artists' acrylic emulsion paints. In: The AIC paintings specialty group postprints: proceedings from the 38th annual meeting of the american institute for conservation and historic works, Milwaukee, Wisconsin, 11-14 May 2010. Washington DC: The American Institute for Conservation of Historic and Artistic Work; 2013. pp. 77-87.

21. Daudin-Schotte M, Bissch M, Joosten I, van Keulen H, van den Berg KJ. Dry cleaning approaches for unvarnished paint surfaces. In: Mecklenburg MF, Charola AE, Koestler RJ, editors. New insights into the cleaning of paintings: proceedings from the cleaning 2010 international conference, Universidad Politécnica de Valencia and Museum Conservation Institute. Washington D.C: Smithsonian Institution Scholarly Press; 2013. pp. 209-20.

22. Chung JY, Ormsby B, Lee J, Burnstock A, Van den Berg KJ. An investigation of options for surface cleaning unvarnished water-sensitive oil paints based on recent developments for acrylic paints. In: ICOM-CC 18th triennial conference preprints, Copenhagen, 4-8 September 2017, ed. J. Bridgland, art. 1302. Paris: International Council of Museums; 2017. pp. $1-12$.

23. Ormsby B, Lee J, Bonaduce I, Lluveras-Tenorio A. Evaluating cleaning systems for use on water sensitive modern oil paints: a comparative study. In: van den Berg KJ, Bonaduce I, Burnstock A, Ormsby B, Scharff M, Carlyle $L$, Heydenreich G, Keune K, editors. Conservation of modern oil paintings. Springer International Publishing; 2019. pp. 11-35.

24. Stavroudis C, Doherty T. The Modular cleaning program in practice: application to acrylic paintings. In: Mecklenburg MF, Charola AE, Koestler RJ, editors. New insights into the cleaning of paintings: proceedings from the cleaning 2010 international conference, Universidad Politécnica de Valencia and Museum Conservation Institute. Washington D.C: Smithsonian Institution Scholarly Press; 2013. pp. 139-45.

25. Stavroudis C. Gels: evolution in practice. In: Angelova LV, Ormsby B, Townsend JH, Wolbers R, editors. Gels in the conservation of art. London: Archetype Publications Ltd; 2017. pp. 209-17.

26. Sorano-Stedman V, Wolbers R, Germond S. The treatment of Barnett Newman's Shining Forth in the MNAM pompidou collection. In: Angelova LV, Ormsby B, Townsend JH, Wolbers R, editors. Gels in the conservation of art. London: Archetype Publications Ltd; 2017. pp. 29-35.

27. Stavroudis C. More from CAPS3: surfactants, silicone-based solvents, and microemulsions, vol. 34. WAAC Newsletter; 2012. pp. 24-7.

28. Angelova LV, Terech P, Natali I, Dei L, Carretti E, Weiss RG. Cosolvent gellike materials from partially hydrolyzed poly(vinyl acetate)s and borax. Langmuir. 2011;27:11671-82.

29. Angelova LV, Berrie BH, De Ghetaldi K, Kerr A, Weiss RG. Partially hydrolyzed poly(vinyl acetate)-borax based gel-like materials for conservation of art: characterization and applications. Stud Conserv. 2015;60:227-44.

30. Angelova LV, Carretti E, Berrie BH, Weiss RG. Poly(vinyl alcohol)-borax "gels": a flexible cleaning option. In: Angelova LV, Ormsby B, Townsend $J H$, Wolbers R, editors. Gels in the conservation of Art. London: Archetype Publications Ltd; 2017. pp. 231-6.

31. Angelova LV, Matarrese C, Fratini E, Weiss RG, Dei L, Carretti E. Chelating agents in aqueous, partially-hydrolyzed, poly(vinyl acetate) dispersions crosslinked with borax. Physicochemical characterization and an application. Colloids and Surfaces A: Physicochemical and Engineering Aspects. 2018;556:61-71.
32. Cremonesi P. Rigid gels and enzyme cleaning. In: Mecklenburg MF, Charola AE, Koestler RJ, editors. New insights into the cleaning of paintings: proceedings from the cleaning 2010 international conference, Universidad Politécnica de Valencia and Museum Conservation Institute. Washington D.C: Smithsonian Institution Scholarly Press; 2013. pp. 179-84.

33. Cremonesi P. Surface cleaning? Yes, freshly grated Agar gel, please. Stud Conserv. 2016;61:362-7.

34. Angelova LV, Ormsby B, Richardson E. Diffusion of water from a range of conservation treatment gels into paint films studied by unilateral NMR. Part I: acrylic emulsion paint. Microchem J. 2016;124:311-20.

35. Cremonesi $P$, Casoli A. Thermo-reversible rigid hydrogels: their properties and action in cleaning. In: Angelova LV, Ormsby B, Townsend JH, Wolbers $\mathrm{R}$, editors. Gels in the conservation of art. London: Archetype Publications Ltd; 2017. pp. 19-27.

36. Kanth A, Singh MP, Satish C. Optimizing the rigidity of gellan and agar gels for cleaning sensitive acrylic emulsion painted surfaces. Int J Conserv Sci. 2018;9:451-62.

37. Chelazzi D, Giorgi R, Baglioni P. Microemulsions, micelles, and functional gels: how colloids and soft matter preserve works of art. Angew Chem Int Ed. 2018;57:7296-303.

38. Baglioni P, Baglioni M, Bonelli N, Chelazzi D, Giorgi R. Smart soft nanomaterials for cleaning. In: Lazzara G, Fakhrullin R, editors. Nanotechnologies and nanomaterials for diagnostic, conservation and restoration of cultural heritage. Amsterdam, Elsevier Science Publishing Co Inc; 2019. pp. 171-204.

39. Mastrangelo R, Montis C, Bonelli N, Tempesti P, Baglioni P. Surface cleaning of artworks: Structure and dynamics of nanostructured fluids confined in polymeric hydrogel networks. Phys Chem Chem Phys. 2017; 19:23762-72.

40. Bonelli N, Poggi G, Chelazzi D, Giorgi R, Baglioni P. Poly(vinyl alcohol)/ poly(vinyl pyrrolidone) hydrogels for the cleaning of art. J Colloid Interface Sci. 2019;536:339-48.

41. Cardaba I, Poggi G, Baglioni M, Chelazzi D, Maguregui I, Giorgi R. Assessment of aqueous cleaning of acrylic paints using innovative cryogels. Microchem J. 2020;152:104311.

42. Mastrangelo R, Chelazzi D, Poggi G, Fratini E, Pensabene Buemi L, Petruzzellis ML, Baglioni P. Twin-chain polymer hydrogels based on poly(vinyl alcohol) as new advanced tool for the cleaning of modern and contemporary art. Proc Natl Acad Sci U S A. 2020;117:7011-20.

43. Daudin-Schotte $M$, van Keulen $H$. Dry cleaning: research and practice. In: van den Berg KJ, Burnstock A, de Keijzer M, Krueger J, Learner T, de Tagle A, Heydenreich G, editors. Issues in contemporary oil paint. Cham: Springer; 2014. pp. 363-72.

44. Veríssimo Mendes B, van den Berg KJ, Megens L, Joosten I, Daudin M. New approaches to surface cleaning of unvarnished contemporary oil paintings - moist sponges and cloths. In: van den Berg KJ, Burnstock A, de Keijzer M, Krueger J, Learner T, de Tagle A, Heydenreich G, editors. Issues in contemporary oil paint. Cham: Springer; 2014. pp. 373-88.

45. Chris Stavroudis. Personal communication. October 11, 2017.

46. Oldring P, Hayward G, editors. Resins for surface coatings. 2nd ed. London: SITA Technology; 1994.

47. Learner TJS. Analysis of modern paints. Los Angeles: Getty Publications; 2004.

\section{Publisher's Note}

Springer Nature remains neutral with regard to jurisdictional claims in published maps and institutional affiliations. 